\title{
An unsteady point vortex method for coupled fluid-solid problems
}

Received: 12 August 2008 / Accepted: 3 February 2009 / Published online: 2 May 2009

(C) The Author(s) 2009. This article is published with open access at Springerlink.com

\begin{abstract}
A method is proposed for the study of the two-dimensional coupled motion of a general sharp-edged solid body and a surrounding inviscid flow. The formation of vorticity at the body's edges is accounted for by the shedding at each corner of point vortices whose intensity is adjusted at each time step to satisfy the regularity condition on the flow at the generating corner. The irreversible nature of vortex shedding is included in the model by requiring the vortices' intensity to vary monotonically in time. A conservation of linear momentum argument is provided for the equation of motion of these point vortices (Brown-Michael equation). The forces and torques applied on the solid body are computed as explicit functions of the solid body velocity and the vortices' position and intensity, thereby providing an explicit formulation of the vortex-solid coupled problem as a set of non-linear ordinary differential equations. The example of a falling card in a fluid initially at rest is then studied using this method. The stability of broadside-on fall is analysed and the shedding of vorticity from both plate edges is shown to destabilize this position, consistent with experimental studies and numerical simulations of this problem. The reduced-order representation of the fluid motion in terms of point vortices is used to understand the physical origin of this destabilization.
\end{abstract}

Keywords Fluid-solid interaction · Point vortex · Vortex shedding

PACS 47.15.ki, 47.63.mc

\section{Introduction}

Complex interaction phenomena can arise from the coupled motions of a solid body and a surrounding fluid, such as the complex trajectories of falling tree leaves or the wind-induced flapping of flags. Fluid-solid interactions are also essential for aerial and aquatic biological locomotion: insects and fishes flap their wings and fins to create around them a flow able to generate the thrust and lift forces necessary for their motion in their environment $[54,56]$. Understanding the physical mechanisms involved in such locomotion schemes is an ongoing engineering challenge to design efficient propulsion solutions inspired by biological locomotion. In all these applications, the motions of the solid body and the fluid are governed by systems of equations of different mathematical nature (Newton's law and the Navier-Stokes equations) and coupled, on moving

Communicated by H. Aref

S. Michelin $(\varangle) \cdot$ S. G. Llewellyn Smith

Department of Mechanical and Aerospace Engineering, Jacobs School of Engineering, UCSD, La Jolla, CA 92093-0411, USA

E-mail: smichelin@ucsd.edu

E-mail:sgls@ucsd.edu

S. Michelin

Ecole Nationale Supérieure des Mines de Paris, 60-62 Boulevard Saint Michel, 75272 Paris Cedex 06, France 
boundaries, through the boundary conditions imposed by the solid motion on the fluid velocity and the forces applied by the fluid on the solid body.

The full numerical simulation of the coupled problem is therefore a computationally complex and expensive problem. It can be carried out using grids fitted on the solid body and a co-moving frame [2,23,41] or using immersed boundary methods $[39,58,59]$. The complexity of direct numerical simulations justifies parallel efforts to develop simplified models able to capture the physical nature of the flow while significantly reducing the computational cost. The present work is such an attempt.

Reduced-order models are based on simplifications in the flow dynamics arising in the particular regime considered. The present work focuses on the two-dimensional motion of a solid body with sharp edges in laminar flows with a relatively high Reynolds number $R e$ (typically $R e \sim 100-1,000$ ). Viscous effects are concentrated in the boundary layers around the solid body. At the sharp edges, these boundary layers separate into free shear layers that quickly roll-up into vortical structures [44], characteristic of high-Re non-turbulent flows over sharp-edged bodies. It has also been observed in numerical simulations that, in this regime, the fluid forces on the solid body are dominated by the aerodynamic pressure and only weakly depend on the Reynolds number (see for example the simulations in [55]).

Several empirical models for the forces created by the flow over an elongated body have been proposed $[1,41,52]$. These models are generally based on added inertia contributions and well-known results for established steady flows past airfoils with circulation. For example, Tanabe and Kaneko [52] identifies the lift on a falling card with that obtained in the Kutta-Joukowski lift theorem for an airfoil in uniform translation at low angle of attack (see the stern criticisms of [35] and the response by [53]). The model used in [1,41] also includes a viscous drag term and a correction to the circulation around the solid body due to its rotation. These models do not include any unsteady effects (terms proportional to the solid acceleration or the vortex shedding rate) other than added inertia. However, they provide a useful tool for problems where the computational cost of direct numerical simulation is prohitive (e.g. optimization problems as in [6]). They also allow to identify the different contributions to the fluid forces $[2,5]$.

The present model belongs to a different category of reduced-order models and retains the full unsteadiness of the flow. Observing that the main influence of viscosity in the regime described above lies in the shedding of vorticity from the sharp edges and that viscous forces on the solid can be neglected as a good first approximation, the flow can be represented using potential flow theory. The shedding of vorticity and the vortex wake are accounted for by introducing manually into the flow a distribution of vortex singularities. The amount of shed vorticity is determined so as to cancel exactly the singularity arising in the potential flow near the shedding corner. Two main approaches have been considered in the literature, depending on whether the shedding of singularities is continuous (vortex sheet) or discontinuous (point vortices). In the former (see for example $[11,24,49])$, a continuous vortex distribution is shed and advected by the flow following the Birkhoff-Rott equation (see for example [45]). The regularity condition is satisfied by the shedding of a new element of the vortex sheet at each time step. In the latter, discrete vortices are shed and the regularity condition is satisfied by adjusting the intensity of the last shed vortex.

The model described here belongs to the second approach and uses the shedding of point vortices with unsteady intensity (Brown-Michael vortices [9]). The intensity of the vortices varies monotonically to represent the irreversible nature of the vortex roll-up. A new vortex is shed when the intensity reaches a maximum, representing a new roll-up in the vortex sheet $[16,20,27]$. This method has been previously applied to study the shedding of vorticity from a finite or semi-infinite plate exposed to a prescribed uniform flow with no rotation $[14,16]$ and compared to continuous shedding methods [15]. The originality of the present work is to generalize the method to arbitrary body shapes and motions and to couple the vortex model to the solid dynamics. The motion of the solid body under the influence of the surrounding fluid can thereby be computed.

This approach neither requires DNS calculations, nor the solution of integro-differential equations as in the vortex sheet method used by $[24,25,49]$. Note that in such vortex sheet calculations (e.g. $[42,51])$ the vortex sheet is modelled as a set of point vortices, and the last vortex (representing the center of the rolled-up spiral) deserves particular treatment. A smoothing kernel must be introduced to avoid numerical issues linked to the ill-posedness of the vortex sheet equation [30]. Furthermore, Pullin [42] and Smith [51] explicitly state that the last vortex and the branch cut must be force-free, which is equivalent to the Brown-Michael vortex model. It is therefore also of interest to understand how a purely Brown-Michael vortex model behaves when coupled with the solid motion.

In Sect. 2, the physical assumptions of the vortex model are presented briefly. In Sect. 3, the equations for the vortex motion and intensity are derived for a general body shape and coupled to the solid body dynamics 
after determining explicit expressions for the forces and torques applied by the fluid on the solid body in terms of the vortex properties. In Sect. 4, the small-time analysis of the equations is performed generalizing the work of [16]. In Sect. 5, this method is applied to study the effect of vorticity shedding on the stability of the fall of a two-dimensional card in a fluid initially at rest. Finally, conlusions are presented in Sect. 6.

\section{Unsteady point vortex model}

\subsection{Description of the point vortex method}

We consider the motion of a solid body with sharp edges in a fluid. The flow around the solid is assumed incompressible, inviscid and initially at rest. According to Kelvin's circulation theorem, it remains irrotational at all time and potential flow theory is used to describe the flow. In the absence of vortex shedding, the presence of sharp edges on the solid body generates an algebraic velocity singularity there. To satisfy the regularity condition at each of the sharp corners, vorticity is introduced in the flow in the form of point vortices with unsteady, monotonically increasing intensity (in magnitude). In the following, we simply refer to these vortices as unsteady point vortices. This method was previously used to study the vortex shedding past a finite or semi-infinite plate with prescribed orthogonal translation [14-16]-the purpose of the present work is to generalize the method to arbitrary motions of a general body, including rotation, and to formulate the fully coupled problem of the fluid and solid motions.

The point vortex, whose intensity is adjusted at each time to cancel exactly the singularity in the velocity field at the generating sharp corner, is a low-order representation of the roll-up of the vortex sheet shed in a real flow from the solid corner by the separation of the boundary layers. In this model, the vorticity is considered concentrated at one point, and the vorticity of the remainder of the sheet is neglected. The reader is referred to [12] for more details. In complex potential flow theory, the point vortex must be connected to the generating corner by a branch cut for the logarithmic point vortex complex potential to be single-valued. The complex velocity is continuous across the branch cut as is the streamfunction (imaginary part of the complex potential). However, the real part of the complex potential is discontinuous and the jump is equal to $\pm \Gamma_{n}$ with $\Gamma_{n}$ the intensity of the corresponding point vortex.

Through the regularity condition, the intensity of the unsteady point vortex is adjusted in time, representing the infinite roll-up of a vortex sheet and the transfer of vorticity from the shedding edge to the vortex core. It is not physically possible to unroll such a vortex sheet since this would correspond to a negative diffusion inside the core. To include this physical argument in the point vortex model, we require that the intensity of the point vortices does not decrease in magnitude. If the intensity of a point vortex reaches an extremum, this vortex will keep a steady intensity later on and a new unsteady vortex is started from the same generating corner to enforce the regularity condition at all time. Graham [20] first discussed this shedding criterion, as well as other possible shedding criteria for point vortices in the flow around a sharp wedge. At any time in the flow past a solid body with $P$ generating corners, $P$ vortices are being shed with unsteady intensity $\Gamma_{1}(t), \ldots, \Gamma_{P}(t)$ to satisfy the regularity of the flow at each of these corners. There may be other vortices present in the flow, which were shed in the past, and whose intensities $\Gamma_{P+1}, \ldots, \Gamma_{N}$ have been frozen.

\subsection{Equation of motion for a point vortex with unsteady intensity}

Several models have been proposed for the motion of point vortices in the presence of solid bodies. In Kirchhoff's equation, the velocity of the point vortex is that of the desingularized background flow and Newton's second law is satisfied everywhere in the fluid except at the position of the vortex itself [31]. However, for an unsteady point vortex, this approach results in an unbalanced force on the branch cut [44]. To satisfy the conservation of momentum around the vortex and the branch cut altogether, this equation needs to be modified. Denoting $\dot{z}_{n}$ the velocity of the point vortex $n$ with position and intensity $\left(z_{n}, \Gamma_{n}\right)$, shed from the corner of (time-varying) position $z_{n, 0}$, we have

$$
\dot{z}_{n}+\left(z_{n}-z_{n, 0}\right) \frac{\dot{\Gamma}_{n}}{\Gamma_{n}}=\overline{\tilde{w}_{n}}
$$

with $\tilde{w}_{n}$ the desingularized complex velocity of the fluid at the position of the vortex. Equation (1) is usually referred to as the Brown-Michael equation [9], although it was in fact first introduced by Edwards [18] in the study of the steady three-dimensional vortex sheet shed by a delta wing. In this steady three-dimensional 
setting, the third dimension plays the role of the time-dependence in (1). It was then proposed by [10] and [44] for unsteady two-dimensional flows and point vortices with unsteady intensity. A derivation of (1) based on a conservation of fluid momentum argument is presented in Appendix A.

\section{Equations of motion of a general sharp-edged solid body}

In this section, the equations of motion for a general solid body with sharp edges shedding unsteady point vortices are derived. The motion of the solid body is studied in the inertial laboratory frame using a fixed system of axes. Let $z$ and $w=u-\mathrm{i} v$ be the complex variables describing respectively the position and velocity in the physical space. Conformal mapping will be used to describe the solid geometry and compute the flow around the body. We write $\zeta$ for the position in the mapped plane.

\subsection{Geometry}

The geometry and motion of the solid body are described by mapping its boundary $\mathcal{C}$ in the physical space from the circle $\mathscr{C}$ of radius $a$ centered at the origin in the mapped plane through the transformation

$$
z=c+\mathrm{e}^{\mathrm{i} \theta} g(\zeta)
$$

where $c$ is the position of the centroid in physical space and $\theta$ denotes the rotation of the solid body compared to a reference configuration (note that the definitions of $c, \theta$ and $g$ are interdependent). This notation is summarized on Fig. 1. The function $g$ maps the outside of $\mathscr{C}$ in the mapped plane onto the outside of the solid body $\mathcal{C}$ in the physical plane. We assume that this mapping is univalent on and outside of $\mathscr{C}$ (each point outside the solid $\mathcal{C}$ corresponds to one and only one point outside $\mathscr{C}$ in the mapped plane). We focus on mappings $g$ of the form

$$
g(\zeta)=\sum_{j=-1}^{M} \frac{G_{j}}{\zeta^{j}}
$$

satisfying the following properties:

- $g^{\prime}$ has no zero outside of $\mathscr{C}$, and a finite number $P$ of zeros on $\mathscr{C}$ itself, denoted $\zeta_{p, 0}=a \mathrm{e}^{\mathrm{i} \phi_{p}}$ with $0 \leq \phi_{p} \leq 2 \pi$ for $1 \leq p \leq P$. We further assume that $g^{\prime \prime}\left(\zeta_{p, 0}\right)$ is non-zero and finite: these points correspond to corners of the solid boundary $\mathcal{C}$ in the physical plane, where both edges of the sharp corner have the same tangential direction.

- $G_{-1}=1$, so that $g(\zeta) \sim \zeta$ at infinity.

- $c$ is the position of the centroid of the body, therefore (see for example [33])

$$
\sum_{k, l=-1, k+l \geq-1}^{M} \frac{l G_{k} G_{l} \bar{G}_{k+l}}{a^{2(k+l)}}=0 .
$$

Only the first of the above conditions is restrictive as the second and third can always be achieved by redefining the parameter $a$ and the variables $\theta$ and $c$. Although we restrict ourselves to solid bodies with flat corners (zero angle), this form of mapping allows to describe a wide variety of solid body shapes with sharp edges.

The area and moment of inertia with respect to the center of mass of this body can be computed as

$$
\left.\mathcal{S}=\frac{1}{2} \operatorname{Im}\left[\int_{\mathscr{C}} \overline{g(\zeta)} g^{\prime}(\zeta) \mathrm{d} \zeta\right]=\pi\left(a^{2}-\sum_{k=1}^{M} \frac{k\left|G_{k}\right|^{2}}{a^{2 k}}\right), \quad I=\frac{\mathcal{M}}{4 \mathcal{S}} \operatorname{Im}\left[\int_{\mathscr{C}} \overline{\left(g(\zeta)^{2}\right.}-g(\zeta)^{2}\right) g(\zeta) g^{\prime}(\zeta) \mathrm{d} \zeta\right]
$$

with $\mathcal{M}$ the mass of the solid body. We assume here that the solid is homogeneous. A complicated expression for $I$ can be obtained explicitly in terms of the coefficients $G_{k}$. 


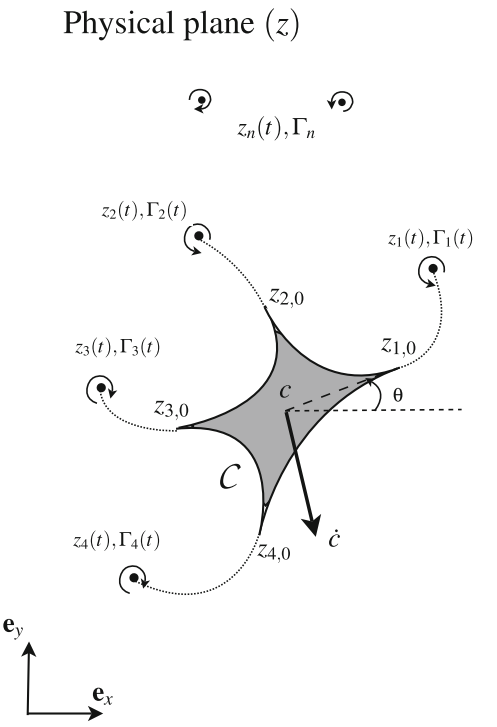

Mapped plane $(\zeta)$

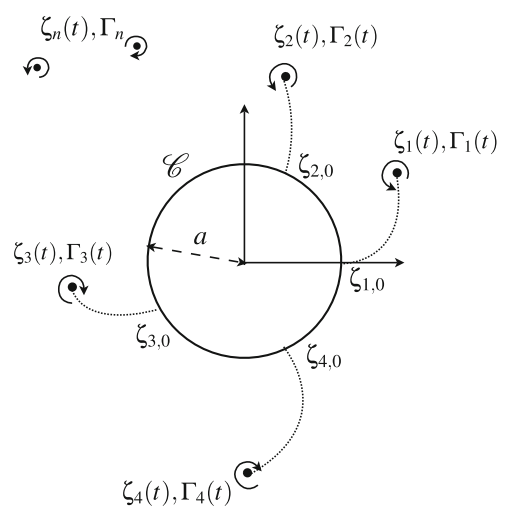

Fig. 1 Notation used for the motion of a general solid body. Left In the physical plane, the position is noted by $z=x+\mathrm{i} y$ and the physical contour of the solid body is $\mathcal{C}$. The position of the center of mass is $c$ and the orientation $\theta$ is measured as the angle between a reference direction in the solid and the horizontal axis. Right The problem is considered in the mapped plane, where positions are noted by $\zeta$. The contour $\mathscr{C}$ is mapped onto $\mathcal{C}$ in the physical space through the mapping (2)

\subsection{Complex potential and velocity around the moving solid body}

The solid body is shedding vortices from its $P$ sharp edges during its motion. At time $t$, we denote by $\zeta_{n}$ the positions of the vortices in the mapped plane, by $z_{n}$ their corresponding positions in the physical plane and by $\Gamma_{n}$ their intensites, with $1 \leq n \leq N$. By convention, vortex $p$ is being shed from corner $p$ corresponding to $\zeta_{p, 0}=a \mathrm{e}^{\mathrm{i} \phi_{p}}$ in the mapped plane $(1 \leq p \leq P)$. The $N-P$ other vortices have a frozen intensity and have been shed and released in the past. Therefore, $\Gamma_{P+1}, \Gamma_{P+2}, \ldots, \Gamma_{N}$ are independent of time while the first $p$ vortices have unsteady intensity $\Gamma_{1}(t), \ldots, \Gamma_{P}(t)$. This convention requires renumbering the vortices when a new vortex is started; $N$ is being incremented and the vortex that was just frozen becomes vortex $N$. We assume that there is no flow and no net circulation at infinity (this is the case for example when the system is started from rest according to Kelvin's circulation theorem).

The complex potential can then be obtained as a linear superposition of the contribution of the motion of the body and of the different vortices:

$$
F=F_{b}+\sum_{n=1}^{N} \Gamma_{n} F_{n}
$$

The potential $F_{b}$ is harmonic outside the solid body and the corresponding velocity decays like $z^{-2}$ at infinity. On the solid boundary $\mathcal{C}$, it satisfies (see for example [17]):

$$
\operatorname{Re}\left[-\mathrm{i} F_{b}\right]=\operatorname{Re}\left[-\mathrm{i} \dot{\bar{c}}(z-c)-\frac{\omega}{2}|z-c|^{2}\right]
$$

with $\omega=\dot{\theta}$, the angular velocity of the solid. This can be easily related to the Schwarz problem on the unit disk and can be solved using the Poisson formula, defining $U=-\dot{\bar{c}} \mathrm{e}^{\mathrm{i} \theta}$,

$$
F_{b}=U(\zeta-g(\zeta))+\frac{a^{2}}{\zeta} \bar{U}-\mathrm{i} \omega r(\zeta), \quad r(\zeta)=\sum_{l=1}^{M+1} \frac{R_{l}}{\zeta^{l}} \quad \text { and } \quad R_{l}=\sum_{k=-1}^{M-l} \frac{\bar{G}_{k} G_{l+k}}{a^{2 k}}, \quad 0 \leq l \leq M+1
$$

Note that the velocity potential due to the rotation $r(\zeta)$ has a dipolar component at infinity unless $R_{1}=$ $\sum G_{l} \bar{G}_{l+1} / a^{2 l}=0$ as investigated in [33]. 
The potential due to each point vortex satisfying the no-flow boundary condition on the solid and the zero-circulation condition at infinity can be obtained in the mapped plane using the circle theorem as:

$$
F_{n}=\frac{1}{2 \pi \mathrm{i}} \log \left[\frac{\zeta-\zeta_{n}}{\zeta-\frac{a^{2}}{\bar{\zeta}_{n}}}\right] .
$$

This definition differs from the one in [38] because the circulation at infinity must be zero here.

Finally, the complex potential $F=\phi+\mathrm{i} \psi$ is obtained by linear superposition, as is the complex velocity $w=u-\mathrm{i} v$ :

$$
\begin{aligned}
& F=U(\zeta-g(\zeta))+\frac{a^{2}}{\zeta} \bar{U}-\mathrm{i} \omega r(\zeta)+\sum_{n=1}^{N} \frac{\Gamma_{n}}{2 \pi \mathrm{i}} \log \left(\frac{\zeta-\zeta_{n}}{\zeta-\frac{a^{2}}{\bar{\zeta}_{n}}}\right), \\
& w=\frac{\mathrm{e}^{-\mathrm{i} \theta}}{g^{\prime}(\zeta)} \frac{\mathrm{d} F}{\mathrm{~d} \zeta}=\frac{\mathrm{e}^{-\mathrm{i} \theta}}{g^{\prime}(\zeta)}\left[U-\frac{a^{2}}{\zeta^{2}} \bar{U}-\mathrm{i} \omega r^{\prime}(\zeta)+\sum_{n=1}^{N} \frac{\Gamma_{n}}{2 \pi \mathrm{i}}\left(\frac{1}{\zeta-\zeta_{n}}-\frac{\bar{\zeta}_{n}}{\zeta \bar{\zeta}_{n}-a^{2}}\right)\right]+\dot{\bar{c}}
\end{aligned}
$$

From (5), the potential $r(\zeta)$ satisfies $\operatorname{Re}\left(r\left(a \mathrm{e}^{\mathrm{i} \phi}\right)\right)=g\left(a \mathrm{e}^{\mathrm{i} \phi}\right) \overline{g\left(a \mathrm{e}^{\mathrm{i} \phi}\right)} / 2$ for $0 \leq \phi \leq 2 \pi$. Differentiating this relation twice with respect to $\phi$, and applying at $\zeta_{p, 0}$, remembering $g^{\prime}\left(\zeta_{p, 0}\right)=0$, leads to the following relations

$$
\operatorname{Im}\left(\zeta_{p, 0} r_{p}^{\prime}\right)=0, \quad \operatorname{Re}\left[\zeta_{p, 0} r_{p}^{\prime}+\zeta_{p, 0}^{2}\left(r_{p}^{\prime \prime}-g_{p}^{\prime \prime} \overline{g_{p}}\right)\right]=0
$$

where we defined for convenience of notation $g_{p}=g\left(\zeta_{p, 0}\right)$ and similarly, $g_{p}^{\prime \prime}, r_{p}^{\prime}$ and $r_{p}^{\prime \prime}$ are the values of the corresponding functions of $\zeta$ when evaluated at $\zeta_{p, 0}$.

\subsection{Regularity condition and motion of the vortices}

The regularity condition at the shedding edges $z_{p, 0}$ imposes that $w$ is finite there. In (7b), the term between brackets must vanish at $\zeta_{p, 0}$ to cancel the singularity introduced by $g^{\prime}$ vanishing at these points. Using $\zeta_{p, 0}=$ $a^{2} / \bar{\zeta}_{p, 0}$, we obtain

$$
2 \operatorname{Im}\left(U \zeta_{p, 0}\right)-\omega \zeta_{p, 0} r_{p}^{\prime}+\sum_{n=1}^{N} \frac{\Gamma_{n}}{2 \pi}\left(1+2 \operatorname{Re}\left[\frac{\zeta_{p, 0}}{\zeta_{n}-\zeta_{p, 0}}\right]\right)=0, \quad 1 \leq p \leq P .
$$

Note from (8) that all terms in (9) are real.

Rewriting the complex potential in (7a) as $F=\tilde{F}_{n}+\frac{\Gamma_{n}}{2 \pi \mathrm{i}} \log \left(\zeta-\zeta_{n}\right)$, the regularized velocity (1) is

$$
\tilde{w}_{n}=\frac{\mathrm{e}^{-\mathrm{i} \theta}}{g^{\prime}\left(\zeta_{n}\right)}\left[\frac{\mathrm{d} \tilde{F}_{n}}{\mathrm{~d} \zeta}\left(\zeta_{n}\right)-\frac{\Gamma_{n}}{4 \mathrm{i} \pi} \frac{g^{\prime \prime}\left(\zeta_{n}\right)}{g^{\prime}\left(\zeta_{n}\right)}\right]
$$

where the last term is known as the Routh correction [11,32]. Using (2), (7b) and (10), the equation of motion for the vortices (1) becomes, in the mapped plane,

$$
\begin{aligned}
g^{\prime}\left(\zeta_{n}\right) \dot{\zeta}_{n}+\left(g\left(\zeta_{n}\right)-g\left(\zeta_{n, 0}\right)\right) \frac{\dot{\Gamma}_{n}}{\Gamma_{n}}= & -\mathrm{i} \omega g\left(\zeta_{n}\right)+\frac{1}{\overline{g^{\prime}\left(\zeta_{n}\right)}}\left[\bar{U}-\frac{a^{2}}{\bar{\zeta}_{n}^{2}} U+\mathrm{i} \omega \overline{r^{\prime}\left(\zeta_{n}\right)}\right. \\
& \left.-\sum_{j \neq n} \frac{\Gamma_{j}}{2 \pi \mathrm{i}}\left(\frac{1}{\bar{\zeta}_{n}-\bar{\zeta}_{j}}-\frac{\zeta_{j}}{\zeta_{j} \bar{\zeta}_{n}-a^{2}}\right)+\frac{\Gamma_{n}}{2 \pi \mathrm{i}}\left(\frac{\zeta_{n}}{\zeta_{n} \bar{\zeta}_{n}-a^{2}}+\frac{\overline{g^{\prime \prime}\left(\zeta_{n}\right)}}{2 \overline{g^{\prime}\left(\zeta_{n}\right)}}\right)\right] .
\end{aligned}
$$




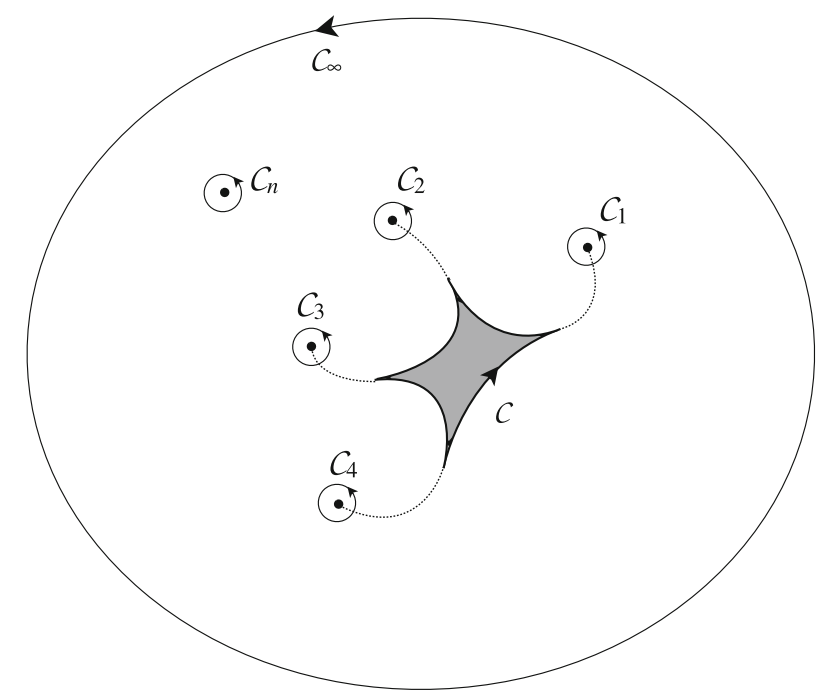

Fig. 2 Details of the contours used in the computation of the integrals in (12) with analytic integrand outside the body except at the position of the vortices. From Cauchy's theorem, the contour integral on $\mathcal{C}$ can be replaced by a contour integral on a circle $\mathcal{C}_{\infty}$ with radius going to infinity, if the contribution from each of the infinitesimally small circular contours $\mathcal{C}_{n}$ encircling each vortex (steady or unsteady) is removed

\subsection{Forces and torques on the solid body}

In this section, explicit expressions are derived for the total force and torque on the body, which are defined as the integral of the pressure forces and torques over the body surface. This definition ensures that the transfer of energy from the fluid to the solid (work of the total force and torque) is the opposite of the transfer of energy from the solid to the fluid (integral along the body of the pressure multiplied by the normal velocity, as identified in the energy conservation equation obtained from Euler's equations). At this point, one should naturally wonder whether the total energy of the system is conserved in such a formulation. However, it is very difficult to define the energy of the system here since the kinetic energy of a fluid with point vortices is infinite. Although the integral of the fluid kinetic energy converges far from the body and vortices due to the absence of net flow or circulation there, each point vortex introduces a logarithmic singularity in the kinetic energy of the flow. We do not attempt here to suggest or prove that the regular part of the kinetic energy should be a conserved quantity. Instead, we focus on the conservation of momentum and angular momentum as both quantities are finite, even for a flow with point vortices.

\subsubsection{Force}

The force on a solid body shedding vortices can be obtained from the results of [46] in terms of complex integrals on the contour of the body:

$$
f=f_{x}+\mathrm{i} f_{y}=\mathrm{i} \rho \sum_{n=1}^{N} z_{n, 0} \dot{\Gamma}_{n}+\overline{\frac{\mathrm{i} \rho}{2} \int_{\mathcal{C}} w^{2} \mathrm{~d} z}+\frac{\mathrm{d}}{\mathrm{d} t}\left[\mathrm{i} \rho \int_{\mathcal{C}} z w \mathrm{~d} z\right]+\rho S \ddot{c}
$$

with $c$ the position of the solid's centroid and $z_{n, 0}$ the position of corner $n$ in the physical plane. The integrands in (12) are analytic functions of $z$ except at the positions of the different vortices. According to Cauchy's theorem, the integration contour can be stretched to infinity without changing the value of the integrals provided that the contribution around each vortex is removed: $\int_{\mathcal{C}}=\int_{\mathcal{C}_{\infty}}-\sum \int_{\mathcal{C}_{n}}$ with $\mathcal{C}_{n}$ an infinitesimally small circle around the $n$-th vortex and $\mathcal{C}_{\infty}$ a circle of radius $R \gg 1$ encircling the solid body and all the vortices at all time (see Fig. 2). On $\mathcal{C}_{\infty}$, the complex potential and complex velocity can be expanded as

$$
F=F_{0}+\frac{a_{1}}{z}+\frac{a_{2}}{z^{2}}+O\left(R^{-3}\right), \quad w=-\frac{a_{1}}{z^{2}}-\frac{2 a_{2}}{z^{3}}+O\left(R^{-4}\right)
$$


and (13) defines the variables $a_{1}$ and $a_{2}$, while near the $n$-th vortex,

$$
w=\frac{\Gamma_{n}}{2 \pi \mathrm{i}\left(z-z_{n}\right)}+\tilde{w}_{n}+O\left(z-z_{n}\right) .
$$

Using (13) and (14) as well as (1), the force on the solid body (12) simplifies to

$$
f=2 \pi \rho \frac{\mathrm{d} a_{1}}{\mathrm{~d} t}+\rho S \frac{\mathrm{d}^{2} c}{\mathrm{~d} t^{2}} .
$$

The expression for $a_{1}$ can be obtained from (2) and (7a). At infinity,

$$
\zeta=z \mathrm{e}^{-\mathrm{i} \theta}-\left(c \mathrm{e}^{-\mathrm{i} \theta}+G_{0}\right)-G_{1} \frac{\mathrm{e}^{\mathrm{i} \theta}}{z}+O\left(R^{-2}\right)
$$

and after expanding (7a) for $\zeta \rightarrow \infty$, we obtain

$$
a_{1}=\left[a^{2} \bar{U}-G_{1} U-\mathrm{i} \omega R_{1}+\sum_{n=1}^{N} \frac{\mathrm{i} \Gamma_{n}}{2 \pi}\left(\zeta_{n}-\frac{a^{2}}{\bar{\zeta}_{n}}\right)\right] \mathrm{e}^{\mathrm{i} \theta},
$$

so that the force (15) becomes

$$
\begin{aligned}
f= & \rho \mathrm{e}^{\mathrm{i} \theta}\left[\left(2 \pi a^{2}-S\right) \dot{\bar{U}}-2 \pi G_{1} \dot{U}-2 \pi \mathrm{i} R_{1} \dot{\omega}+\mathrm{i} \omega\left(2 \pi a^{2}-S\right) \bar{U}-2 \pi \mathrm{i} \omega G_{1} U+2 \pi R_{1} \omega^{2}\right. \\
& \left.+\mathrm{i} \frac{\mathrm{d}}{\mathrm{d} t} \sum_{n=1}^{N} \Gamma_{n}\left(\zeta_{n}-\frac{a^{2}}{\bar{\zeta}_{n}}\right)-\omega \sum_{n=1}^{N} \Gamma_{n}\left(\zeta_{n}-\frac{a^{2}}{\bar{\zeta}_{n}}\right)\right] .
\end{aligned}
$$

\subsubsection{Barycentric torque on the solid body}

The computation by [46] of the torque applied by the fluid on the body about a fixed point can be extended easily to the case of a point moving in the laboratory frame. If this point is taken to be the centroid of the solid body (or equivalently its center of mass since we will consider here only homogeneous bodies), the torque simplifies further into:

$$
\mathcal{T}^{*}=\frac{\rho}{2} \sum_{n=1}^{N}\left|z_{n, 0}-c\right|^{2} \dot{\Gamma}_{n}+\frac{\rho}{2} \operatorname{Re}\left[2 \dot{\bar{c}} \int_{\mathcal{C}}(z-c) w \mathrm{~d} z-\int_{\mathcal{C}}(z-c) w^{2} \mathrm{~d} z+\frac{\mathrm{d}}{\mathrm{d} t} \int_{\mathcal{C}}|z-c|^{2} w \mathrm{~d} z\right] .
$$

The integrands are analytic functions of $z$ except for the last integral $A=\int_{\mathcal{C}}|z-c|^{2} w \mathrm{~d} z$. However, on the contour of the solid body $\mathcal{C}$,

$$
|z-c|^{2}=g(\zeta) \overline{g(\zeta)}=\left(\sum_{k=-1}^{M} G_{k} \zeta^{-k}\right)\left(\sum_{j=-1}^{M} \frac{\bar{G}_{j} \zeta^{j}}{a^{2 j}}\right)=R_{0}+\sum_{l=1}^{M+1}\left(\frac{R_{l}}{\zeta^{l}}+\frac{\bar{R}_{l} \zeta^{l}}{a^{2 l}}\right)
$$

with the definition of $R_{l}$ in (6) for $0 \leq l \leq M+1$. Using the previous equation as well as (7b), one obtains:

$$
A=\int_{\mathscr{C}}\left[R_{0}+\sum_{l=1}^{M+1}\left(\frac{R_{l}}{\zeta^{l}}+\frac{\bar{R}_{l} \zeta^{l}}{a^{2 l}}\right)\right]\left[U \sum_{k=1}^{M} \frac{k G_{k}}{\zeta^{k+1}}-\bar{U} \frac{a^{2}}{\zeta^{2}}+\mathrm{i} \omega \sum_{l=1}^{M} \frac{l R_{l}}{\zeta^{l+1}}+\sum_{n=1}^{N} \frac{\Gamma_{n}}{2 \pi \mathrm{i}}\left(\frac{1}{\zeta-\zeta_{n}}-\frac{\bar{\zeta}_{n}}{\zeta \bar{\zeta}_{n}-a^{2}}\right)\right] \mathrm{d} \zeta .
$$

Using Cauchy's theorem, the previous integral can be computed as

$$
A=-\sum_{n=1}^{N} \Gamma_{n}\left[R_{0}+\sum_{l=1}^{M+1}\left(\frac{R_{l}}{\zeta_{n}^{l}}+\frac{\bar{R}_{l}}{\bar{\zeta}_{n}^{l}}\right)\right]-2 \pi \omega \sum_{l=1}^{M+1} \frac{l\left|R_{l}\right|^{2}}{a^{2 l}}+2 \pi \mathrm{i}\left(-\overline{R_{1} U}+U \sum_{l=1}^{M} \frac{l G_{l} \bar{R}_{l}}{a^{2 l}}\right)
$$


and from the centroid condition (3), we also have

$$
\sum_{l=1}^{M} \frac{l G_{l} \bar{R}_{l}}{a^{2 l}}=R_{1}
$$

Hence, finally,

$$
A=-\sum_{n=1}^{N} \Gamma_{n}\left[R_{0}+2 \operatorname{Re}\left(r\left(\zeta_{n}\right)\right)\right]-2 \pi \omega \sum_{l=1}^{M+1} \frac{l\left|R_{l}\right|^{2}}{a^{2 l}}-4 \pi \operatorname{Im}\left(R_{1} U\right) .
$$

Note that $A$ is purely real.

Considering the other integrals in (18), the integrands are analytic except at the position of the different vortices, and we can therefore apply the same technique used for the force in the previous section by stretching the integration contour to infinity and removing the contribution of the infinitesimally small contours around each vortex. Doing so, we obtain for the torque:

$$
\mathcal{T}^{*}=\frac{\rho}{2} \sum_{n=1}^{N}\left|z_{n, 0}-c\right|^{2} \dot{\Gamma}_{n}+\rho \operatorname{Re}\left[\sum_{n=1}^{N} \Gamma_{n}\left(\tilde{w}_{n}-\dot{\bar{c}}\right)\left(z_{n}-c\right)-2 \pi \mathrm{i} a_{1} \dot{\bar{c}}\right]+\frac{\rho \dot{A}}{2},
$$

with $a_{1}$ and $A$ respectively defined in (16) and (19). Using (1), and $g\left(\zeta_{n}\right)=\left(z_{n}-c\right) \mathrm{e}^{-\mathrm{i} \theta}, \mathcal{T}^{*}$ can be rewritten

$$
\mathcal{T}^{*}=\frac{\rho}{2} \sum_{n=1}^{N}\left[\dot{\Gamma}_{n}\left|g\left(\zeta_{n}\right)-g\left(\zeta_{n, 0}\right)\right|^{2}+\frac{\mathrm{d}}{\mathrm{d} t}\left(\Gamma_{n}\left|g\left(\zeta_{n}\right)\right|^{2}\right)\right]-2 \pi \rho \operatorname{Im}\left[U a_{1} \mathrm{e}^{-\mathrm{i} \theta}\right]+\frac{\rho \dot{A}}{2} .
$$

Using (16) and (19), the barycentric torque is finally obtained as

$$
\begin{aligned}
\mathcal{T}^{*}= & \frac{\rho}{2} \sum_{n=1}^{N}\left\{\dot{\Gamma}_{n}\left|g\left(\zeta_{n}\right)-g\left(\zeta_{n, 0}\right)\right|^{2}+\frac{\mathrm{d}}{\mathrm{d} t}\left[\Gamma_{n}\left(\left|g\left(\zeta_{n}\right)\right|^{2}-R_{0}-2 \operatorname{Re}\left(r\left(\zeta_{n}\right)\right)\right)\right]-2 \Gamma_{n} \operatorname{Re}\left[U\left(\zeta_{n}-\frac{a^{2}}{\bar{\zeta}_{n}}\right)\right]\right\} \\
& +2 \pi \rho \operatorname{Im}\left[G_{1} U^{2}\right]+2 \pi \rho \omega \operatorname{Re}\left[R_{1} U\right]-\pi \rho \dot{\omega} \sum_{l=1}^{M+1} \frac{l\left|R_{l}\right|^{2}}{a^{2 l}}-2 \pi \rho \operatorname{Im}\left[R_{1} \dot{U}\right]
\end{aligned}
$$

\subsubsection{Conservation of total momentum and comparison with the case of steady vortices}

It is enlightening to compare the expressions (15) and (20) for the motion of a solid body shedding unsteady point vortices to the situation where the solid body does not shed vorticity but moves in a flow with pre-existing point vortices of steady intensity. Ramodanov [43] derived the equations of motion of a cylinder and a single point vortex with steady intensity. It was also shown that the equations of motion of a two-dimensional solid body and $N$ point vortices with steady intensity are Hamiltonian $[8,26,47,48]$.

From [26], the force $\mathbf{f}_{s}$ and barycentric torque $\mathcal{T}_{s}^{*}$ on the solid body in the case of an inviscid flow with point vortices of steady intensity are obtained as

$$
\begin{gathered}
\mathbf{f}_{s}=-\frac{\mathrm{d} \mathbf{p}_{\text {fluid }}}{\mathrm{d} t} \text { with } \mathbf{p}_{\text {fluid }}=\rho \int_{C} \mathbf{x} \times(\mathbf{n} \times \mathbf{u}) \mathrm{d} s+\rho \sum_{k=1}^{N} \Gamma_{k} \mathbf{x}_{k} \times \mathbf{e}_{3}, \\
\mathcal{T}_{s}^{*} \mathbf{e}_{3}=\frac{\rho}{2} \frac{\mathrm{d}}{\mathrm{d} t}\left(\sum_{k=1}^{N} \Gamma_{k}\left\|\mathbf{x}_{k}-\mathbf{c}\right\|^{2}\right) \mathbf{e}_{3}-\dot{\mathbf{c}} \times \mathbf{p}_{\text {fluid }}+\frac{\rho}{2} \frac{\mathrm{d}}{\mathrm{d} t} \int_{\mathcal{C}}\|\mathbf{x}-\mathbf{c}\|^{2}(\mathbf{n} \times \mathbf{u}) \mathrm{d} s
\end{gathered}
$$

with $\mathbf{x}_{k}$ the position vector of vortex $k$ and $\mathbf{p}_{\text {fluid }}$ the fluid linear momentum.

In (15), $2 \pi a_{1}=\mathrm{i} \int_{\mathcal{C}_{\infty}} z w \mathrm{~d} z$ and using the identity (see for example [46])

$$
\int_{\mathcal{C}} z \mathrm{~d} \psi=\frac{\mathrm{d} S c}{\mathrm{~d} t}
$$


the expression (15) derived for the force in the case of shedding of vortices with unsteady intensity can be rewritten $f=-\dot{p}_{\text {fluid }}$ with $p_{\text {fluid }}=-\mathrm{i} \rho \int_{\mathcal{C}} z \mathrm{~d} \phi-\mathrm{i} \rho \sum \Gamma_{k} z_{k}$ with $\phi$ the real part of the complex potential. This is equivalent (in complex notation) to the expression (22a) obtained from the Hamiltonian system used in [26]. As in the derivation by [21] who obtained a result similar to (15) for a fixed solid, the form of the equation of motion for the point vortices (1) is essential as it allows one to cancel exactly any unbalanced force on the branch cut introduced by the shedding of a point vortex with unsteady intensity (see Appendix A). The momentum is conserved in the fluid in an integral sense around the vortex and the branch cut, which explains the similarity with the result of the Hamiltonian theory in [26].

Following a similar approach and transferring (22) to complex notation, we observe that the three last terms on the right-hand side of (20) correspond to the three terms on the right-hand side of (22) in the same order. The shedding of unsteady point vortices introduces an additional term $\mathcal{T}_{v}$ in the barycentric torque applied on the solid proportional to the product of the rate of change of each vortex intensity by the square of the distance between that vortex and the generating corner.

A careful application of the conservation of angular momentum in an integral sense around the vortex and branch cut, similar to the one detailed in Appendix A for the conservation of linear momentum, would show that, because of the form of (1), an unbalanced torque remains on the branch cut equal to the opposite of the additional term $\mathcal{T}_{v}$. Our analysis is therefore consistent with other studies on complex potential flows.

The existence of this additional term is inherent to the vortex model chosen here. Because of the limited number of degrees of freedom available for the position of the point vortex (its intensity is fixed by the regularity condition), it is not possible to satisfy at the same time the conservation of linear and angular momentum around the vortex and branch cut. The Brown-Michael vortex is in this regard an improvement over the Kirchhoff vortex that does not satisfy either momentum conservation law if the vortex has unsteady intensity. A possible alternative approach would consist in satisfying another conservation law (for example angular momentum conservation) and accepting the existence of an unbalanced force on the branch cut [22].

\subsubsection{Added inertia contributions and momentum transfer to the vortices}

In (17) and (21), the terms not involving $\Gamma_{n}$ are added mass terms. For the general shape considered here, the added-mass coefficients can be recovered from (17) and (21). Considering the reference directions 1 and 2 of added mass theory to coincide with the horizontal and vertical axes when $\theta=0$, then the $3 \times 3$ symmetric added-mass tensor $\mathscr{M}_{i j}$ with $i, j=1,2,6$ is obtained as (see for example [40])

$$
\mathscr{M}=\rho\left[\begin{array}{ccc}
2 \pi\left[a^{2}+\operatorname{Re}\left(G_{1}\right)\right]-S & -2 \pi \operatorname{Im}\left(G_{1}\right) & -2 \pi \operatorname{Im}\left(R_{1}\right) \\
-2 \pi \operatorname{Im}\left(G_{1}\right) & 2 \pi\left[a^{2}-\operatorname{Re}\left(G_{1}\right)\right]-S & 2 \pi \operatorname{Re}\left(R_{1}\right) \\
-2 \pi \operatorname{Im}\left(R_{1}\right) & 2 \pi \operatorname{Re}\left(R_{1}\right) & \pi \sum \frac{l\left|R_{l}\right|^{2}}{a^{2 l}}
\end{array}\right]
$$

The terms proportional to $\Gamma_{n}$ or $\dot{\Gamma}_{n}$ are additional forces and torques resulting from the shedding of vorticity. Using the results of Sect.3.4.3, they can be divided into two kinds: those arising from the transfer of angular momentum and linear momentum from the solid to the fluid as explained in [48] and an additional torque, corresponding to an additional transfer of angular momentum because of the vortex representation chosen here.

\subsection{Coupled equations of motion}

We assume here that an external force $\mathcal{F}(t)$ and torque $\mathcal{T}(t)$ are applied on the solid body. The system of equations for the coupled vortex-solid problem is:

$$
\begin{gathered}
\mathcal{M} \ddot{c}=\mathcal{F}(t)+\rho \mathrm{e}^{\mathrm{i} \theta}\left[\left(2 \pi a^{2}-S\right) \dot{\bar{U}}-2 \pi G_{1} \dot{U}-2 \pi \mathrm{i} R_{1} \dot{\omega}+\mathrm{i} \omega\left(2 \pi a^{2}-S\right) \bar{U}-2 \pi \mathrm{i} \omega G_{1} U+2 \pi R_{1} \omega^{2}\right. \\
\left.\quad+\mathrm{i} \frac{\mathrm{d}}{\mathrm{d} t} \sum_{n=1}^{N} \Gamma_{n}\left(\zeta_{n}-\frac{a^{2}}{\bar{\zeta}_{n}}\right)-\omega \sum_{n=1}^{N} \Gamma_{n}\left(\zeta_{n}-\frac{a^{2}}{\bar{\zeta}_{n}}\right)\right]
\end{gathered}
$$




$$
\begin{gathered}
I \dot{\omega}=\mathcal{T}(t)+2 \pi \rho \operatorname{Im}\left[G_{1} U^{2}\right]+2 \pi \rho \omega R e\left[R_{1} U\right]-\pi \rho \dot{\omega} \sum_{l=1}^{M+1} \frac{l\left|R_{l}\right|^{2}}{a^{2 l}}-2 \pi \rho \operatorname{Im}\left[R_{1} \dot{U}\right] \\
+\frac{\rho}{2} \sum_{n=1}^{N}\left[\dot{\Gamma}_{n}\left|g\left(\zeta_{n}\right)-g\left(\zeta_{n, 0}\right)\right|^{2}+\frac{\mathrm{d}}{\mathrm{d} t}\left[\Gamma_{n}\left(\left|g\left(\zeta_{n}\right)\right|^{2}-R_{0}-2 \operatorname{Re}\left(r\left(\zeta_{n}\right)\right)\right]-2 \Gamma_{n} \operatorname{Re}\left[U\left(\zeta_{n}-\frac{a^{2}}{\bar{\zeta}_{n}}\right)\right]\right]\right. \\
g^{\prime}\left(\zeta_{n}\right) \dot{\zeta}_{n}+\left(g\left(\zeta_{n}\right)-g\left(\zeta_{n, 0}\right)\right) \frac{\dot{\Gamma}_{n}}{\Gamma_{n}}=-\mathrm{i} \omega g\left(\zeta_{n}\right)+\frac{1}{g^{\prime}\left(\zeta_{n}\right)}\left[\bar{U}-\frac{a^{2}}{\bar{\zeta}_{n}^{2}} U+\mathrm{i} \omega \overline{r^{\prime}\left(\zeta_{n}\right)}\right. \\
\left.-\sum_{j \neq n} \frac{\Gamma_{j}}{2 \pi \mathrm{i}}\left(\frac{1}{\bar{\zeta}_{n}-\bar{\zeta}_{j}}-\frac{\zeta_{j}}{\zeta_{j} \bar{\zeta}_{n}-a^{2}}\right)+\frac{\Gamma_{n}}{2 \pi \mathrm{i}}\left(\frac{\zeta_{n}}{\zeta_{n} \bar{\zeta}_{n}-a^{2}}+\frac{\overline{g^{\prime \prime}\left(\zeta_{n}\right)}}{2 \overline{g^{\prime}\left(\zeta_{n}\right)}}\right)\right] \\
2 \operatorname{Im}\left(U \zeta_{p, 0}\right)-\omega \zeta_{p, 0} r_{p}^{\prime}+\sum_{n=1}^{N} \frac{\Gamma_{n}}{2 \pi}\left(1+2 R e\left[\frac{\zeta_{p, 0}}{\zeta_{n}-\zeta_{p, 0}}\right]\right)=0, \quad 1 \leq p \leq P
\end{gathered}
$$

with $U=-\dot{\bar{c}} \mathrm{e}^{\mathrm{i} \theta}$. At time $t$, the number of real unknowns is $2 N+P+3$ (position and orientation of the solid body ( 3 unknowns), position of the vortices ( $2 N$ unknowns) and intensity of the vortices being presently shed from each corner ( $P$ unknowns), the other intensities having been frozen to a known value). The system (24) is a system of $2 N+P+3$ equations (2 from Newton's second law (24a), 1 from the conservation of angular momentum (24b), $2 N$ for the position of the vortices (24c) and $P$ from the regularity condition at each corner $(24 d))$.

\section{Small-time behavior and vortex shedding}

The system is started from rest at $t=0$ and evolves from this rest position because of an external force (e.g. gravity) or torque. The motion of the sharp edges induces the shedding of vortices from each corner. When a new vortex is started from a generating corner, the right-hand side of the equation of motion (24c) of this vortex is singular as the vortex and its image conjugate are at the same point. The distance between the generating corner and the vortex in the mapped plane varies like a fractional power of time, consistently with the singular behavior at $t=0$. The following section uses the same approach as in [13,16], whose results are thereby extended to arbitrary body shapes and motions. We focus on the vortex shedding and, as in [16], assume first that the solid motion is known. The initial behavior of the solid body can then be obtained from the small-time analysis of $(24 a-b)$.

\subsection{Shedding of the first vortices}

We first consider the case of the first vortex shed from each of the $P$ generating corners when the motion of the solid is started. We assume that initially $U \sim U_{0} t^{\alpha}$ and $\omega \sim \omega_{0} t^{\beta}$.

Defining the reduced variable $\eta_{p}$ such that $\zeta_{p}=\zeta_{p, 0}\left(1+\eta_{p}\right)$ and keeping only the dominant terms in (24c) and (24d), we obtain for $1 \leq p \leq P$

$$
\begin{gathered}
2 \operatorname{Im}\left(U \zeta_{p, 0}\right)-\omega \zeta_{p, 0} r_{p}^{\prime}+\frac{\Gamma_{p}}{\pi} \operatorname{Re}\left(\frac{1}{\eta_{p}}\right)=0 \\
g_{p}^{\prime \prime} \eta_{p} \dot{\eta}_{p}+\frac{g_{p}^{\prime \prime} \eta_{p}^{2}}{2} \frac{\dot{\Gamma}_{p}}{\Gamma_{p}}=\frac{1}{a^{4} \overline{g_{p}^{\prime \prime}} \bar{\eta}_{p}}\left[-2 \operatorname{iIm}\left(U \zeta_{p, 0}\right)+\mathrm{i} \omega \overline{\zeta_{p, 0} r_{p}^{\prime}}-\frac{\mathrm{i} \Gamma_{p}}{2 \pi}\left(\frac{1}{\eta_{p}+\bar{\eta}_{p}}+\frac{1}{2 \bar{\eta}_{p}}\right)\right],
\end{gathered}
$$

as the contribution of vortices $j \neq p$ to the regularity condition at corner $p$ and to the induced velocity on vortex $p$ are negligible. Remembering that $\zeta_{p, 0} r_{p}^{\prime}$ is real [see (8)], we can define the real quantity 


$$
\begin{gathered}
F_{p}(t)=2 \operatorname{Im}\left(U \zeta_{p, 0}\right)-\omega \zeta_{p, 0} r_{p}^{\prime} \sim F_{p 0} t^{\mu} \text {, with } \mu=\min (\alpha, \beta) \text { and obtain } \\
\Gamma_{p}=-\frac{2 \pi \eta_{p} \bar{\eta}_{p}}{\eta_{p}+\bar{\eta}_{p}} F_{p}(t) \\
\eta_{p} \dot{\eta}_{p}+\frac{\eta_{p}^{2}}{2} \frac{\dot{\Gamma}_{p}}{\Gamma_{p}}=-\frac{\mathrm{i} F_{p}(t)}{a^{4}\left|g_{p}^{\prime \prime}\right|^{2} \bar{\eta}_{p}}\left(1-\frac{\eta_{p}\left(3 \bar{\eta}_{p}+\eta_{p}\right)}{2\left(\eta_{p}+\bar{\eta}_{p}\right)^{2}}\right) .
\end{gathered}
$$

If we decompose the complex number $\eta_{p}$ into its polar representation, we obtain a system of equations identical to the one derived by [13] for the shedding of Brown-Michael point vortices from a semi-infinite plate with imposed motion. While it is intuitively reasonable, as the initial shedding is expected to be driven by the local behavior of the flow, the present approach generalizes the results to arbitrary bodies with both translation and rotation. Using the same method as in [13], we obtain that initially

$$
\begin{gathered}
z_{p}-z_{p, 0}=\frac{\zeta_{p, 0}^{2} g_{p}^{\prime \prime} \mathrm{e}^{\mathrm{i} \theta}}{2} \eta_{p}^{2}, \quad \eta_{p}=\mathrm{e}^{-\mathrm{i} \frac{\pi}{4} \operatorname{sgn}\left(F_{p 0}\right)}\left[\frac{\left|F_{p 0}\right|}{a^{4}(2 \mu+1)\left|g_{p}^{\prime \prime}\right|^{2} \sqrt{2}}\right]^{1 / 3} t^{(\mu+1) / 3}, \\
\Gamma_{p}=-\operatorname{sgn}\left(F_{p 0}\right) \pi\left[\frac{2 F_{p 0}^{4}}{(2 \mu+1) a^{4}\left|g_{p}^{\prime \prime}\right|^{2}}\right]^{1 / 3} t^{(4 \mu+1) / 3} .
\end{gathered}
$$

Here, $\eta_{p}^{2}$ is purely imaginary and $g_{p}^{\prime \prime} \zeta_{p, 0}^{2} \mathrm{e}^{\mathrm{i} \theta}$ is the orientation of the local tangent. Hence, the first vortices are shed orthogonally to the shedding corner.

We have shown that if $U$ and $\omega$ initially have power-law behaviors, $t^{\alpha}$ and $t^{\beta}$, the circulation of the vortices behaves like $t^{(4 \mu+1) / 3}$ and the distance to the shedding corner like $t^{2(\mu+1) / 3}$, where $\mu=\min (\alpha, \beta)$. Taking this information into account, we observe that in (24a) and (24b) the added mass terms initially dominate the contribution of the vortices. Under prescribed external forces and torques, the initial motion of the solid body is driven by added inertia. The influence of vortex shedding only becomes important later on. This is particularly convenient for the solution of this problem as it allows one to solve for the initial solid motion using added mass theory and then use the result for $U$ and $\omega$ to determine the initial behavior of the vortices.

\subsection{Shedding of subsequent vortices}

When a vortex reaches a maximum intensity at $t=t_{s}$, its intensity is frozen and a new vortex is started from the corresponding shedding corner to satisfy the regularity condition there. The vortices are also reindexed so as to keep the first $P$ vortices be the ones with unsteady intensity. The equation of motion for the new vortex needs to be solved analytically at small time as the right-hand side of (24c) displays an initial singularity as for the first vortices. Without loss of generality, we assume that the new vortex is shed from corner 1 . This new vortex is labeled 1 while the vortex that was just frozen becomes vortex $N$. Because $\dot{\Gamma}_{N}=0$ and is continuous at $t=t_{s}$, the velocity of all vortices $2 \leq j \leq N$ are continuous as well as their intensity and so is the velocity and rotation rate of the solid body.

As before, we define $\eta$ so that $\zeta_{1}=\zeta_{1,0}(1+\eta)$. Substitution in (24d) leads to:

$$
2 \operatorname{Im}\left(U \zeta_{1,0}\right)-\omega \zeta_{1,0} r_{1}^{\prime}+\frac{\Gamma_{1}}{\pi} \operatorname{Re}\left(\frac{1}{\eta}\right)+\sum_{n=2}^{N} \frac{\Gamma_{n}}{2 \pi}\left[1+2 \operatorname{Re}\left(\frac{\zeta_{1,0}}{\zeta_{n}-\zeta_{1,0}}\right)\right]=0
$$

We assume that $\eta \sim \tau^{\delta}, \Gamma_{1} \sim \tau^{\gamma}$ (with $\tau=t-t_{s}$ and $t_{s}$ the time of shedding) and that $\operatorname{Re}(\eta$ ) is not initially negligible compared to $|\eta|$; by dominant balance in the previous equation, enforcing the regularity condition at $t=t_{s}^{ \pm}$, we obtain $\gamma=\delta+2$. Then, the effect of the new vortex in the right-hand side of (24c) is negligible and by dominant balance $\delta=1 / 2$ and $\gamma=5 / 2$. The equation of motion (24c) simplifies, keeping only the dominant terms and using $(24 \mathrm{~d})$,

$$
\zeta_{1,0}^{2} g_{1}^{\prime \prime}\left(\eta \dot{\eta}+\frac{5 \eta^{2}}{4 \tau}\right)=-\mathrm{i} \omega g_{1}+\frac{1}{\overline{g_{1}^{\prime \prime} \zeta_{1,0}^{2}}}\left[2 U \zeta_{1,0}+\mathrm{i} \omega \overline{r_{1}^{\prime \prime} \zeta_{1,0}^{2}}+\sum_{n=2}^{N} \frac{\mathrm{i} \Gamma_{n}}{2 \pi}\left(\frac{\zeta_{k}^{2} \bar{\zeta}_{1,0}^{2}}{\left(\zeta_{j} \zeta_{1,0}-a^{2}\right)^{2}}-\frac{\bar{\zeta}_{1,0}^{2}}{\left(\bar{\zeta}_{1,0}-\bar{\zeta}_{n}\right)^{2}}\right)\right]
$$


where all quantities other than $\eta$ are evaluated at $\tau=0$. The right-hand side of the previous equation is $O\left(\tau^{0}\right)$, and corrections to that approximation would only add terms of order $\tau$ initially negligible.

$$
\text { Using (8), (9) and } \zeta_{1,0} \bar{\zeta}_{1,0}=a^{2} \text {, }
$$

$$
a^{4}\left|g_{1}^{\prime \prime}\right|^{2}\left(\eta \dot{\eta}+\frac{5 \eta^{2}}{4 \tau}\right)=2 \operatorname{Re}\left(U \zeta_{1,0}\right)+\mathrm{i} \omega \overline{\left[\zeta_{1,0} r_{1}^{\prime}-\bar{g}_{1} g_{1}^{\prime \prime} \zeta_{1,0}^{2}+r_{1}^{\prime \prime} \zeta_{1,0}^{2}\right]}-\sum_{2}^{N} \frac{\Gamma_{n}}{\pi} \operatorname{Im}\left(\frac{\zeta_{1,0} \zeta_{n}}{\left(\zeta_{n}-\zeta_{1,0}\right)^{2}}\right)
$$

This can be integrated for $\eta$ as

$$
\eta^{2}=C \tau, \quad \text { with } C=\frac{4}{7 a^{4}\left|g_{1}^{\prime \prime}\right|^{2}}\left[2 \operatorname{Re}\left(U \zeta_{1,0}\right)+\mathrm{i} \omega\left[\overline{\zeta_{1,0} r_{1}^{\prime}-\bar{g}_{1} g_{1}^{\prime \prime} \zeta_{1,0}^{2}+r_{1}^{\prime \prime} \zeta_{1,0}^{2}}\right]-\sum_{2}^{N} \frac{\Gamma_{n}}{\pi} \operatorname{Im}\left(\frac{\zeta_{1,0} \zeta_{n}}{\left(\zeta_{n}-\zeta_{1,0}\right)^{2}}\right)\right] .
$$

From (8), we observe that $C$, and therefore $\eta^{2}$, are purely real. In physical space,

$$
z_{1}-z_{1,0}=\frac{C}{2} \mathrm{e}^{\mathrm{i} \theta} g_{1}^{\prime \prime} \zeta_{1,0}^{2} \tau
$$

and the vortex is shed parallel to the local tangent at the shedding corner whose direction is given by $\mathrm{e}^{\mathrm{i} \theta} g_{1}^{\prime \prime} \zeta_{1,0}^{2}$. This result extends to arbitrary motions and shapes the result obtained by [16] for a semi-infinite plate in pure translation. It is consistent with the physical analysis of [20]: at the time of shedding, the vortices already present cancel the normal background flow through the regularity condition and the flow is therefore dominated by the tangential component at the corner, which carries the newly shed vortex parallel to the tangential direction. Note that we restrict ourselves here to the case of flat corners (zero angle). In the case of a finite angle, the direction of shedding depends on the direction of the flow at the corner [20].

\subsection{Favorable and unfavorable shedding conditions}

If $C>0, \eta$ is purely real and the vortex goes out of the solid body. However, if $C<0, \eta$ is purely imaginary and this violates the initial assumption of this analysis that $\operatorname{Re}(\eta)$ is not negligible compared to $\operatorname{Im}(\eta)$. Carrying out the small-time analysis to the next order shows that $\zeta_{1}$ is located on the circle $\mathscr{C}$. Hence, in the physical plane, the vortex is pushed by the flow onto the solid boundary $\mathcal{C}$. This is problematic as the vortex and its image would be at the same position and this introduces a singularity in the equations. The case $C<0$ is of the same nature as the limitations to the vortex sheet method presented in [24] and [25]. In those works, it is observed that the integration fails if a newly shed Lagrangian element of the vortex sheet is forced to remain on the solid boundary by the outside flow. Our numerical simulations with prescribed motions confirm the observations by [24] that this problem occurs preferentially at low angles of attack when $\operatorname{Re}\left(U \zeta_{1,0}\right)$ is negative, but that it can also occur at high angles of attack, when the solid body is forced to interact with its own wake. Cortelezzi and Leonard [16] considered the occurrence of such an event to be the result of a numerical error. This does not contradict our analysis as the situations considered numerically by [16] involved the motion of a plate normal to itself with no reversal of the direction of motion, so that vortices are always carried away from the plate. In that case, $C<0$ does not occur.

We refer to the case $C>0$ (resp. $C<0$ ) as favorable (resp. unfavorable) shedding conditions. The case $C<0$ is the main limitation of the present method. It is expected that a purely inviscid approach cannot resolve this issue, as physically in such a situation of shedding at low angle of attack, the new vortex is expected to remain trapped near the shedding edge and interact with the boundary layer, a situation that is not described well by an inviscid model. However, because of the discrete shedding mechanism used here, the occurrence of $C<0$ is only problematic when a new vortex is being shed.

\section{Example: stability of the broadside-on fall of a two dimensional flat plate}

In this section, we use the method outlined previously for a general solid body to study the particular case of a falling two-dimensional flat plate. This problem, known as Maxwell's problem [36], has been extensively 

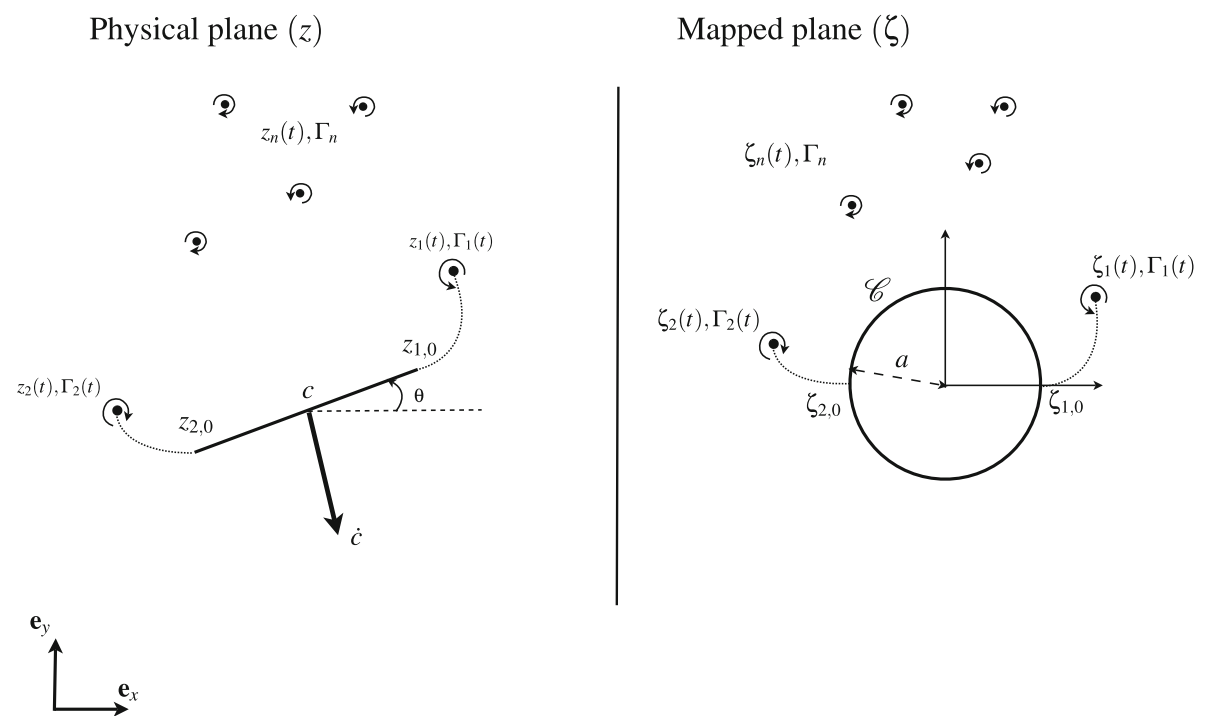

Fig. 3 Notation of Fig. 1 adapted for the particular case of a falling card. The position of the plate's center of mass is noted $c$ and the orientation $\theta$ is the angle between the plate and the horizontal axis. In the mapped plane, the two shedding corners $\zeta_{1,0}$ and $\zeta_{2,0}$ are the intersections of the circle $\mathscr{C}$ with the horizontal axis

studied experimentally for objects such as falling disks [19,57] and long falling plates $[3,4,50]$. Depending on $R e$ and the relative inertia of the fluid and solid, different regimes have been observed. Defining $R e$ based on the solid's chord or width, the typical terminal velocity and the fluid viscosity, it was observed that, below a critical $R e_{c}$ (typically $R e_{c} \sim 200$ ), the fall in the broadside-on (horizontal) position is stable. Initial non-zero angles lead to viscously dampened fluttering oscillations [57]. At higher Re, the dampened regime is not observed. From a non-horizontal initial orientation, three regimes are typically observed: periodic fluttering, periodic tumbling or chaotic [19,50]. More recently two-dimensional numerical simulations were performed for a falling ellipse using moving fitted grids [2,23]. Using the inviscid approach described in the present paper, we study as an example the effect of vortex shedding on the stability of the broadsideon fall. The experimental and numerical results at large Re predict a destabilization of the broadside-on fall $[19,50,57]$.

A homogeneous plate of half-length $l$, mass $\mathcal{M}$ and negligible thickness is released from rest at $t=0$ with an initial angle to the horizontal $\theta_{0}\left(-\pi / 2 \leq \theta_{0} \leq \pi / 2\right)$, and falls under the effect of gravity so that $\mathcal{F}(t)=-\mathrm{i} \mathcal{M} g$ and $\mathcal{T}(t)=0$. Its moment of inertia is $I=\mathcal{M} l^{2} / 3$. The center of mass of the plate is initially at the origin of a fixed system of axes in the inertial laboratory frame. In the following, all quantities are non-dimensionalized using the half-length $l$ of the plate, the density of the fluid $\rho$ and gravity $g$ as reference scales. Two non-dimensional parameters characterize the problem: $\theta_{0}$, the initial release angle, and the ratio of solid and fluid inertia identified as the Froude number in [25]: $\mathrm{Fr}=\mathcal{M} / 2 \rho l^{2}$.

\subsection{Equations of motion}

\subsubsection{Coupled equations for the vortex-plate system}

The plate of unit half-length is obtained using the particular mapping:

$$
g(\zeta)=\zeta+\frac{a^{2}}{\zeta}
$$

with $a=1 / 2$. In the framework of the present paper, all the coefficients $G_{j}$ are zero except for $G_{-1}=1$ and $G_{1}=a^{2}=1 / 4$. From (6), we obtain that all $R_{j}$ are equal to zero except for $R_{0}=2 a^{2}=1 / 2$ and $R_{2}=a^{4}=1 / 16$. The solid body has two sharp corners, $\phi_{1}=0$ and $\phi_{2}=\pi$, corresponding to $\zeta_{1,0}=g_{1}=a$ and $\zeta_{2,0}=g_{2}=-a$, where $g_{1}^{\prime \prime}=2 / a=4$ and $g_{2}^{\prime \prime}=-2 / a=-4$ (see Fig. 3). The complex potential (7a) 
and velocity (7b) simplify to

$$
\begin{gathered}
F=-\frac{2 \mathrm{i} a^{2} \operatorname{Im}(U)}{\zeta}-\frac{\mathrm{i} \omega a^{4}}{\zeta^{2}}+\sum_{n=1}^{N} \frac{\Gamma_{n}}{2 \pi \mathrm{i}} \log \left(\frac{\zeta-\zeta_{n}}{\zeta-\frac{a^{2}}{\bar{\zeta}_{n}}}\right), \\
w=\frac{\zeta^{2} \mathrm{e}^{-\mathrm{i} \theta}}{\zeta^{2}-a^{2}}\left[U-\frac{a^{2}}{\zeta^{2}} \bar{U}+\frac{2 \mathrm{i} \omega a^{4}}{\zeta^{3}}+\sum_{n=1}^{N} \frac{\Gamma_{n}}{2 \pi \mathrm{i}}\left(\frac{1}{\zeta-\zeta_{n}}-\frac{\bar{\zeta}_{n}}{\zeta \bar{\zeta}_{n}-a^{2}}\right)\right]+\dot{\bar{c}}
\end{gathered}
$$

where, as before, $\dot{c}=-\bar{U} \mathrm{e}^{\mathrm{i} \theta}$. The plate is infinitely thin so $S=0$ and there is no buoyancy effect. The only force externally applied on the plate is gravity. In non-dimensional form, the system of coupled equations for the motion of the vortices and the plate is obtained from (24)

$$
\begin{aligned}
2 F r(\ddot{c}+\mathrm{i})= & \mathrm{e}^{\mathrm{i} \theta}\left[-\mathrm{i} \pi \operatorname{Im}(\dot{U})+\pi \omega \operatorname{Im}(U)+\mathrm{i} \frac{\mathrm{d}}{\mathrm{d} t} \sum_{n=1}^{N} \Gamma_{n}\left(\zeta_{n}-\frac{a^{2}}{\bar{\zeta}_{n}}\right)-\omega \sum_{n=1}^{N} \Gamma_{n}\left(\zeta_{n}-\frac{a^{2}}{\bar{\zeta}_{n}}\right)\right], \\
\left(\frac{2 F r}{3}+\frac{\pi}{8}\right) \dot{\omega}= & \pi \operatorname{Im}[U] \operatorname{Re}[U]+\frac{1}{2} \sum_{n=1}^{N}\left\{\dot{\Gamma}_{n} \frac{\left|\zeta_{n}-\zeta_{n, 0}\right|^{4}}{\left|\zeta_{n}\right|^{2}}-2 \Gamma_{n} \operatorname{Re}\left[U\left(\zeta_{n}-\frac{a^{2}}{\bar{\zeta}_{n}}\right)\right]\right. \\
& \left.+\frac{\mathrm{d}}{\mathrm{d} t}\left[\Gamma_{n}\left(\zeta_{n} \bar{\zeta}_{n}-a^{2}\right)\left(1+\frac{a^{2}}{\zeta_{n}^{2}}+\frac{a^{2}}{\bar{\zeta}_{n}^{2}}-\frac{a^{2}}{\zeta_{n} \bar{\zeta}_{n}}\right)\right]\right\} \\
\left(1-\frac{a^{2}}{\zeta_{n}^{2}}\right) \dot{\zeta}_{n}+ & \frac{\left(\zeta_{n}-\zeta_{n, 0}\right)^{2}}{\zeta_{n}} \frac{\dot{\Gamma}_{n}}{\Gamma_{n}}=-\mathrm{i} \omega\left(\zeta_{n}+\frac{a^{2}}{\zeta_{n}}\right)+\frac{\bar{\zeta}_{n}^{2}}{\bar{\zeta}_{n}^{2}-a^{2}}\left[\bar{U}-\frac{a^{2}}{\bar{\zeta}_{n}^{2}} U-\frac{2 \mathrm{i} \omega a^{4}}{\bar{\zeta}_{n}^{3}}\right. \\
- & \left.\sum_{j \neq n} \frac{\Gamma_{j}}{2 \pi \mathrm{i}}\left(\frac{1}{\bar{\zeta}_{n}-\bar{\zeta}_{j}}-\frac{\zeta_{j}}{\zeta_{j} \bar{\zeta}_{n}-a^{2}}\right)+\frac{\Gamma_{n}}{2 \pi \mathrm{i}}\left(\frac{\zeta_{n}}{\zeta_{n} \bar{\zeta}_{n}-a^{2}}+\frac{a^{2}}{\bar{\zeta}_{n}\left(\bar{\zeta}_{n}^{2}-a^{2}\right)}\right)\right] \\
& \pm \operatorname{Im}(U)+\omega a+\sum_{n=1}^{N} \frac{\Gamma_{n}}{4 \pi a}\left(1 \pm 2 \operatorname{Re}\left[\frac{a}{\zeta_{n} \mp a}\right]\right)=0 .
\end{aligned}
$$

The system (30) is a complete system of ordinary differential equations for the plate's position $c$ and orientation $\theta$, the vortices positions $z_{n}$ (or equivalently $\zeta_{n}$ in the mapped plane) and the two unknown intensities $\Gamma_{1}$ and $\Gamma_{2}$.

The limit case $F r=0$ deserves some additional comment. In that case, the left-hand side of (30a) vanishes while the left-hand side of (30b) does not. However, on the right-hand side of (30a) added-mass terms depend on $\ddot{c}$ through $\dot{U}$ and therefore (30a) does not degenerate into an algebraic equation. Furthermore, in the case $F r=0$, the forcing gravity term disappears, and the solution of (30) is trivial, the body does not move and no vortex is shed.

\subsubsection{Small-time behavior}

We have shown in Sect. 4.1 that initially, the effects of vortex shedding are negligible compared to added-inertia. Retaining only added-inertia terms, by dominant balance in (30a) and (30b), we obtain that $\dot{c}$ and therefore $U$ are initially linear functions of time while $\omega \sim t^{3}$. The small-time behavior of the falling card is obtained from (30a) and (30b) defining $c=x_{c}+\mathrm{i} y_{c}$ :

$$
\dot{\omega}=O\left(t^{2}\right), \quad \ddot{x}_{c}=-\frac{\pi \sin 2 \theta_{0}}{2(\pi+2 F r)}+O(t), \quad \ddot{y}_{c}=-1+\frac{\pi \cos ^{2} \theta_{0}}{\pi+2 F r}+O(t)
$$

which is equivalent to the results of [25], since in both the point vortex and the vortex sheet methods, the initial behavior of the falling card is dominated by added mass effects. The initial acceleration of the plate makes an angle $\theta_{f}$ with the vertical $\left(\tan \theta_{f}=\ddot{x} / \ddot{y}\right.$ ) which depends on both $\theta_{0}$ and $\operatorname{Fr}$ (see Fig. 4). For all $F r$, the plate falls vertically if $\theta_{0}=0$ (broadside-on) or $\theta_{0}= \pm \pi / 2$ (edge-on). For large $\operatorname{Fr}$ (heavy plate), the plate's initial fall is almost vertical $\left(\left|\theta_{f}\right| \ll 1\right)$ as the inertia of the fluid is negligible compared to that of the solid. For small 

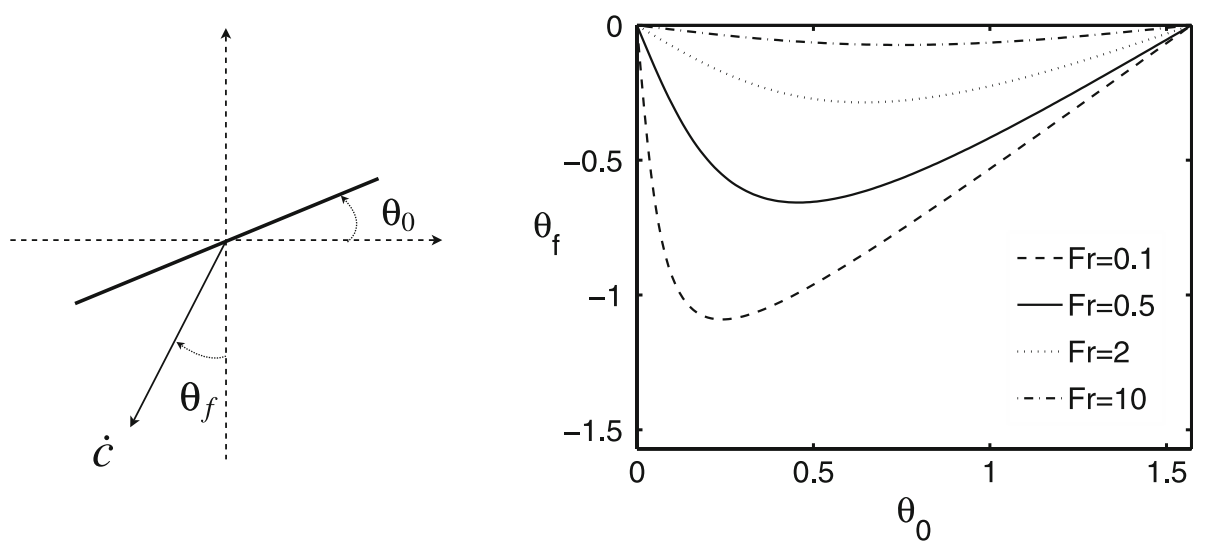

Fig. 4 Left When the plate is released with an initial orientation $\theta_{0}$, its initial velocity and acceleration make an angle $\theta_{f}$ with the vertical axis. Note that $\theta_{f}<0$ if $\theta_{0}>0$. Right Variations of $\theta_{f}$ with $\theta_{0}$ for different values of $F r$. The case $\theta_{0}<0$ is obtained by symmetry

$\operatorname{Fr}$ (light plate), the plate tends to slide initially parallel to itself $\left(\theta_{f} \sim \theta_{0}-\pi / 2\right)$ in order to reduce the relative flow normal to the plate that induces the largest added inertia.

Then, we obtain from (31) and $U=-\dot{\bar{c}} \mathrm{e}^{\mathrm{i} \theta}$ that initially

$$
U \sim U_{0} t, \quad \text { with } U_{0}=\left(\frac{\pi \sin \theta_{0}}{\pi+2 F r}-\frac{2 \mathrm{i} F r \mathrm{e}^{\mathrm{i} \theta_{0}}}{\pi+2 F r}\right) t
$$

From the results of Sect.4.1, the initial motion of the two vortices is obtained as

$z_{1}-\left(c+\mathrm{e}^{\mathrm{i} \theta_{0}}\right)=z_{2}-\left(c-\mathrm{e}^{\mathrm{i} \theta_{0}}\right)=\frac{\mathrm{ie}^{\mathrm{i} \theta_{0}} t^{4 / 3}}{2}\left[\frac{2 F r \cos \theta_{0}}{3 \sqrt{2}(\pi+2 F r)}\right]^{2 / 3}, \quad \Gamma_{1}=-\Gamma_{2}=4 \pi t^{5 / 3}\left[\frac{F r^{4} \cos ^{4} \theta_{0}}{6(\pi+2 F r)^{4}}\right]^{1 / 3}$.

The trajectories of the vortices relative to the plate are initially symmetric as their initial motion is dominated by the normal flow at the shedding corners.

\subsubsection{Numerical solution}

The system of ODEs (30) is solved using the variable time-step Matlab ODE solver ode45. The integration is done analytically using (31) and (32) over the first time step $\Delta t$ (typically $10^{-5}$ ) because the solver cannot handle directly the singular initial behavior of (30c). The event tracking function is used to detect when $\dot{\Gamma}_{1}$ or $\dot{\Gamma}_{2}$ vanishes at which point the integration is stopped, the corresponding vortex intensity becomes frozen, the vortices are reindexed accordingly and a new vortex is started. As for the first vortices, the system is integrated by hand for the first time step, using the results of Sect. 4.2 whose main result (28) becomes in the particular case studied here

$$
\zeta_{1} \sim \zeta_{1,0}(1+\eta), \quad \text { with } \eta^{2}=\frac{4 \tau}{7}\left[2 \operatorname{Re}(U)-\sum_{n=2}^{N} \frac{\Gamma_{n}}{\pi} \operatorname{Im}\left(\frac{\zeta_{n}}{\left(\zeta_{n}-\zeta_{1,0}\right)^{2}}\right)\right] \text {. }
$$

As discussed in Sect. 4.3, $\eta^{2}$ is purely real, and depending on its sign, leads to favorable or unfavorable shedding conditions. In the former case, a new vortex is started successfully and the integration is carried on further using the ODE solver. In the latter case, the integration stops since the shedding of the new vortex cannot be handled using an inviscid vortex method. 


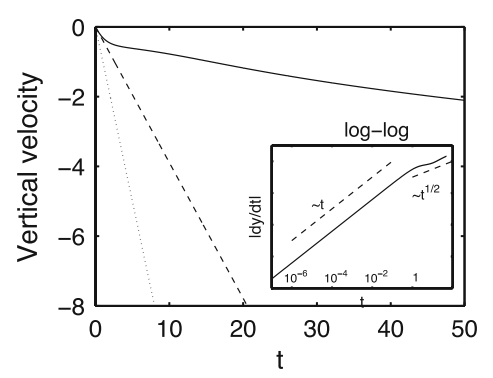

(a)

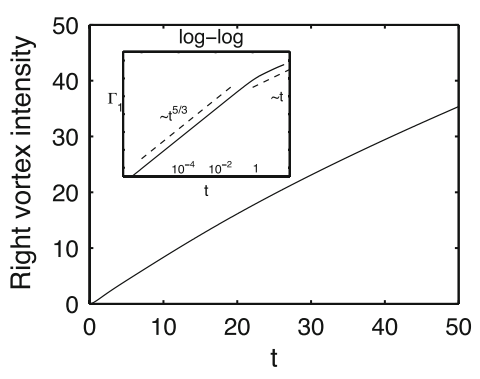

(b)

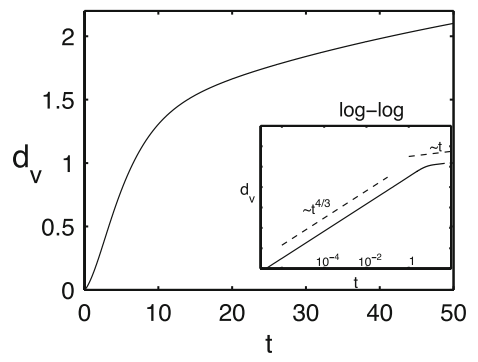

(c)

Fig. 5 Broadside-on fall of a card $\left(\theta_{0}=0\right)$ for $F r=1$. a Vertical velocity of the plate: full vortex shedding model (solid), added inertia only (dashed) and fall in vacuum (dotted). b Intensity of the right vortex. $\mathbf{c}$ Vertical distance $d_{v}$ between the vortex pair and the plate. In each plot, the insert gives a logarithmic plot of the corresponding quantity obtained with the full vortex shedding model to identify scalings

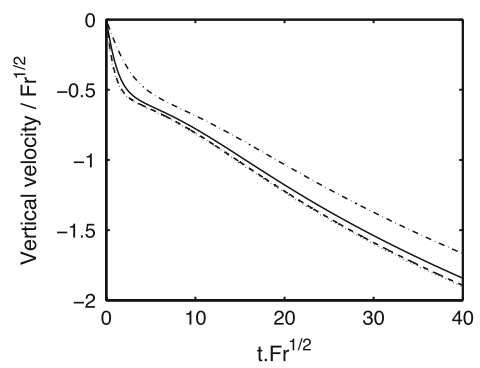

(a)

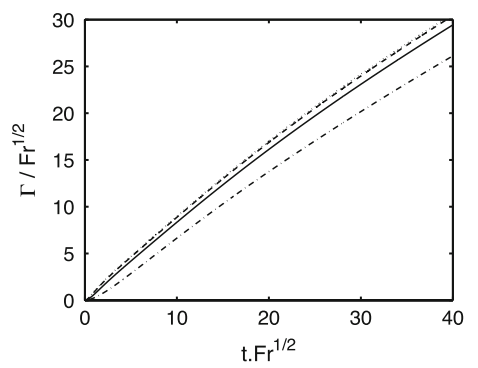

(b)

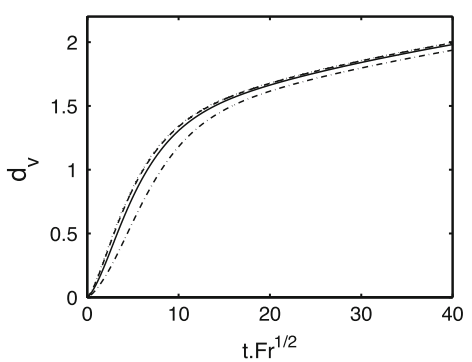

(c)

Fig. 6 Scalings of the broadside-on fall with $F r$ : a the vertical velocity of the plate, b the intensity of the vortices and $\mathbf{c}$ the vertical distance between the vortices and the plate are plotted for $F r=0.05$ (dotted), $F r=0.2$ (dashed), $F r=1$ (solid) and $F r=4$ (dash-dotted), against a rescaled time $t \sqrt{F r}$. The different quantities (velocity, circulation) are rescaled accordingly

\subsection{Fall of a horizontal card}

When the card is released initially with $\theta_{0}=0$, the problem is symmetric with respect to the vertical axis, and this symmetry remains at all time. The card falls vertically with $\theta=0$ (broadside-on). One vortex is shed from each corner with opposite intensities and symmetric positions with respect to the vertical axis. The intensity of these vortices never reaches an extremum so no new vortex is shed. We denote by $d_{v}=\operatorname{Im}\left(z_{1}-c\right)=\operatorname{Im}\left(z_{2}-c\right)$ the vertical distance between the plate and the vortex pair.

Both fluid effects (added inertia and vortex shedding) tend to slow down the plate (see Fig. 5a) as they both correspond to a transfer of negative vertical momentum to the fluid. Figure 5 allows us to identify different phases in the broadside-on fall. During the first phase $(t<1$ for $F r=1)$, the motion of the plate is dominated by added inertia and gravity. The distance $d_{v}$ and intensity $\Gamma_{1}$ follow the scaling laws obtained in (32). After a transition phase $(1<t<15)$, where the vortex shedding influence becomes important and the plate acceleration quickly decreases, a long time regime is achieved in which the fluid forces almost balance gravity. Although the plate dynamics does not seem to follow an exact power-law, its vertical velocity behaves almost like $\sqrt{t}$. The vortex wake intensity grows linearly in time and its distance to the plate varies like $t^{1 / 4}$. This distance varies much slower than the plate vertical position, so the vortex pair seems trapped in the wake of the falling card.

As in [25], when redefining the time scale such that $t^{\prime}=t \sqrt{F r}$ and rescaling the other quantities accordingly, the time evolution of the different quantities for different values of $F r$ collapse onto similar evolution curves (see Fig. 6). With this new reference time-scale, the evolution of the system is almost independent of $F r$. Note that the agreement is best at low $F r$ (typically $F r<1$ ). 


\subsection{Comparison with added mass analysis}

Before studying the behavior of a falling card released with a non-zero angle, we present a brief summary of the solid motion obtained by representing the fluid effects with added inertia only. This also will be useful in the remaining sections to emphasize the effect of vortex shedding. In this section only, the fluid forces and torques are entirely given by the added inertia contribution. Viscosity and vorticity are both neglected. The equations of motion can be obtained from added-mass theory [40] or directly from (30) by removing the contribution of the vortices:

$$
\begin{gathered}
\left(\frac{2 F r}{\pi}+\sin ^{2} \theta\right) \ddot{x}_{c}-\frac{1}{2} \sin 2 \theta \ddot{y}_{c}=\dot{\theta}\left(\dot{y}_{c} \cos 2 \theta-\dot{x}_{c} \sin 2 \theta\right), \\
-\frac{1}{2} \sin 2 \theta \ddot{x}_{c}+\left(\frac{2 F r}{\pi}+\cos ^{2} \theta\right) \ddot{y}_{c}=-\frac{2 F r}{\pi}+\dot{\theta}\left(\dot{y}_{c} \sin 2 \theta+\dot{x}_{c} \cos 2 \theta\right), \\
\left(\frac{1}{8}+\frac{2 F r}{3 \pi}\right) \ddot{\theta}=\left(\frac{\dot{x}_{c}^{2}-\dot{y}_{c}^{2}}{2}\right) \sin 2 \theta-\dot{x}_{c} \dot{y}_{c} \cos 2 \theta .
\end{gathered}
$$

From (34) or its equivalent in a system of axes attached to the plate, an equation for the orientation $\theta$ only can be obtained [34]

$$
\left(\frac{1}{8 F r}+\frac{2}{3 \pi}\right) \ddot{\theta}+\frac{t^{2} \sin 2 \theta}{(\pi+2 F r)}=0, \quad \text { with } \dot{\theta}(0)=0 \text { and } \theta(0)=\theta_{0}
$$

and the motion of the plate's center of mass is recovered from $\theta$ using

$$
\dot{x}_{c}=-\frac{\pi t \sin 2 \theta}{2(\pi+2 F r)}, \quad \dot{y}_{c}=-\frac{t}{\pi+2 F r}\left(2 F r+\pi \sin ^{2} \theta\right) .
$$

The solution of (35) has an oscillatory behavior with decreasing amplitude as confirmed by the WKB solution valid for small initial angle $\theta_{0}$ and $t \gg 1$ :

$$
\theta(t) \sim \frac{C_{0}}{\sqrt{t}} \cos \left(t^{2} \sqrt{\frac{12 \pi F r}{(\pi+2 F r)(3 \pi+16 F r)}}+\phi_{0}\right),
$$

with $C_{0}$ and $\phi_{0}$, two constants obtained by matching with the initial behavior. Exploring the parameter space $\left(\theta_{0}, F r\right)$ shows that, in two dimensions, added inertia only produces a fluttering behavior with algebraically decreasing amplitude and linearly increasing frequency. This is consistent with other theoretical studies that showed that $\theta=0$ is a stable position and $\theta= \pm \pi / 2$ an unstable position [28,34]. Adding asymmetric viscous drag can generate other regimes like tumbling [29,34]. See also [7] for a more complete review of the asymptotic behavior of a falling solid body in an ideal fluid with no shed vorticity.

For large $\mathrm{Fr}$ (heavy plate), the solid inertia is dominant and the motion tends to a pure translation (oscillation in $\theta$ with zero frequency). For small $F r$, the forcing on the solid occurs over a very long time scale $\mathrm{Fr}^{-1 / 2}$ and the motion of the plate is negligible unless $t \gg 1$. The oscillation frequency grows the fastest when both the solid and fluid inertia are comparable, emphasizing the fluid-solid interaction nature of the fluttering motion.

These results show that an infinite-Re model based exclusively on added-inertia (therefore neglecting both viscosity and vorticity shedding) fails to explain the destabilization of the broadside-on fall observed in high-Re experiments. The shedding of vorticity from the plate edges is therefore essential to understand the behavior of the falling plate when viscosity is neglected.

\subsection{Stability of broadside-on fall}

We now consider the case of a card released with a small non-zero initial angle $\theta_{0}$ using the vortex shedding model. A wide range of the parameter space $\left(\theta_{0}, F r\right)$ was explored, leading to the following result: a card initially released with a small non-zero initial angle undergoes fluttering oscillations with growing amplitude 
(see Fig. 7). The broadside-on fall position is therefore unstable ${ }^{1}$; this is consistent with the results obtained using the full vortex sheet representation [25].

We are interested in the development of the instability and how it is influenced by the two non-dimensional parameters $\mathrm{Fr}$ and $\theta_{0}$. Figure 7 shows the behavior of the falling card for $\theta_{0}=\pi / 1,024$ and increasing values of $\mathrm{Fr}$. In all three experiments, new vortices are shed according to the shedding criterion described in the previous sections (three or four in the cases presented in Fig. 7). The wake is dominated by a pair of strong counter-rotating vortices, whose primary effect is to slow down the falling card. The asymmetry introduced by the fluttering motion of the plate is clearly visible on the plot of the streamlines. The plate undergoes a fluttering motion with growing amplitude as can be seen in the variation of its orientation and horizontal position (Fig. 7).

\subsubsection{Scaling of the instability development}

During this growing fluttering motion, the orientation angle is observed to oscillate within an exponential envelope (Fig. 8). We can define the growth rate $\sigma$ of the instability characterizing the exponential envelope and a pseudoperiod $\tau$ for the oscillations. Figure 8 shows the influence of $F r$ and $\theta_{0}$ on the evolution of $\theta$. We observe that the variations of $\theta / \theta_{0}$ are independent of $\theta_{0}$ during the growth regime. The influence of $\theta_{0}$ becomes important when $\theta$ reaches $O(1)$ values and the exponential growth saturates. The Froude number $\mathrm{Fr}$ is observed to influence the pseudoperiod of the oscillations. However, the growth rate of the instability (that determines the shape of the exponential envelope on Fig. 8a) seems independent of $\mathrm{Fr}$.

This qualitative analysis is confirmed by the quantitative results of Fig. 9. Focusing on the successive extrema of the orientation $\theta_{m}$, we observe that for all $F r,\left|\theta_{m}\right| \sim \theta_{0} \mathrm{e}^{\sigma t}$ with $\sigma=0.23$ (Fig. 9a). The pseudoperiod $\tau$ of the oscillations is obtained using the successive occurrences of $\theta=0$ and is plotted on Fig. 9b against $\mathrm{Fr}$, showing a slow decrease of the pseudo-period with $\mathrm{Fr}$, and $\tau \sim \mathrm{Fr}^{-0.12}$. This very weak dependence in $\mathrm{Fr}$ is consistent with the observations of [25], when the different variables are rescaled appropriately to match the notation in [25].

\subsubsection{Characteristics of the growing-amplitude fluttering regime}

During this phase, the vertical motion is at leading order identical to the broadside-on solution obtained for $\theta_{0}=0$. The horizontal position experiences oscillations with the same characteristics $\sigma$ and $\tau$ as the $\theta$-oscillations. The oscillations of $\theta$ and $x_{c}$ are however not in phase: the maximum horizontal deviation is reached slightly before the maximum orientation angle (Fig. 7).

During this fluttering regime, the vortex wake is dominated by a pair of counter-rotating vortices as in the case $\theta=0$. The asymmetry of the wake is characterized by a net circulation around the solid body of $\Gamma_{b}=-\left(\Gamma_{1}+\Gamma_{2}\right)$ and a difference between the vertical positions of the two vortices of $\delta_{v}=\operatorname{Im}\left(z_{1}-z_{2}\right)$. For small $\theta_{0}, \Gamma_{b}$ and $\delta_{v}$ remain small during the initial fluttering regime.

The dominant motion is vertical, and for non-zero $\theta$, the angle of attack at one edge (leading edge) is slightly smaller than at the other (trailing edge). The vorticity shedding rate is expected to be slightly larger at the leading edge, and the corresponding vortex remains closer to the shedding edge because of the BrownMichael corrective term in (1) that tends to pull the vortex back to its shedding corner. This corrective term represents the faster roll-up of a more intense vortex sheet on itself, keeping the center of vorticity closer from the shedding corner. An asymmetry in the vertical velocities of the vortices is therefore introduced and the leading edge vortex moves downward faster with the plate than the other vortex. For $\theta>0$, the left vortex (negative) grows faster than the right vortex and $\dot{\Gamma}_{b}>0$ and $\dot{\delta}_{v}>0$. We indeed observe that $\theta, \dot{\Gamma}_{b}$ and $\dot{\delta}_{v}$ are approximately in phase throughout the oscillation (Fig. 10a). Because of the small phase difference between the horizontal and angular motions, the two vortices have about the same vertical position $\left(\delta_{v} \sim 0\right)$ and opposite intensities $\left(\Gamma_{b} \sim 0\right)$ when the plate horizontal velocity changes sign (Fig. 10b).

\subsubsection{Destabilization and transfer of momentum to the vortices}

A physical interpretation of this destabilization is obtained by considering the fluid effect on the solid body. In this section, we will successively consider the transfer of momentum (linear or angular) and of kinetic energy

\footnotetext{
${ }^{1}$ We understand here by instability that for any small value of the initial angle (initial values $\theta_{0}$ as low as $\pi / 2^{15}$ were tested), the plate is observed to move away from the broadside-on equilibrium. We do not attempt to provide a mathematical proof of the instability of the time-dependent solution of equations (30).
} 

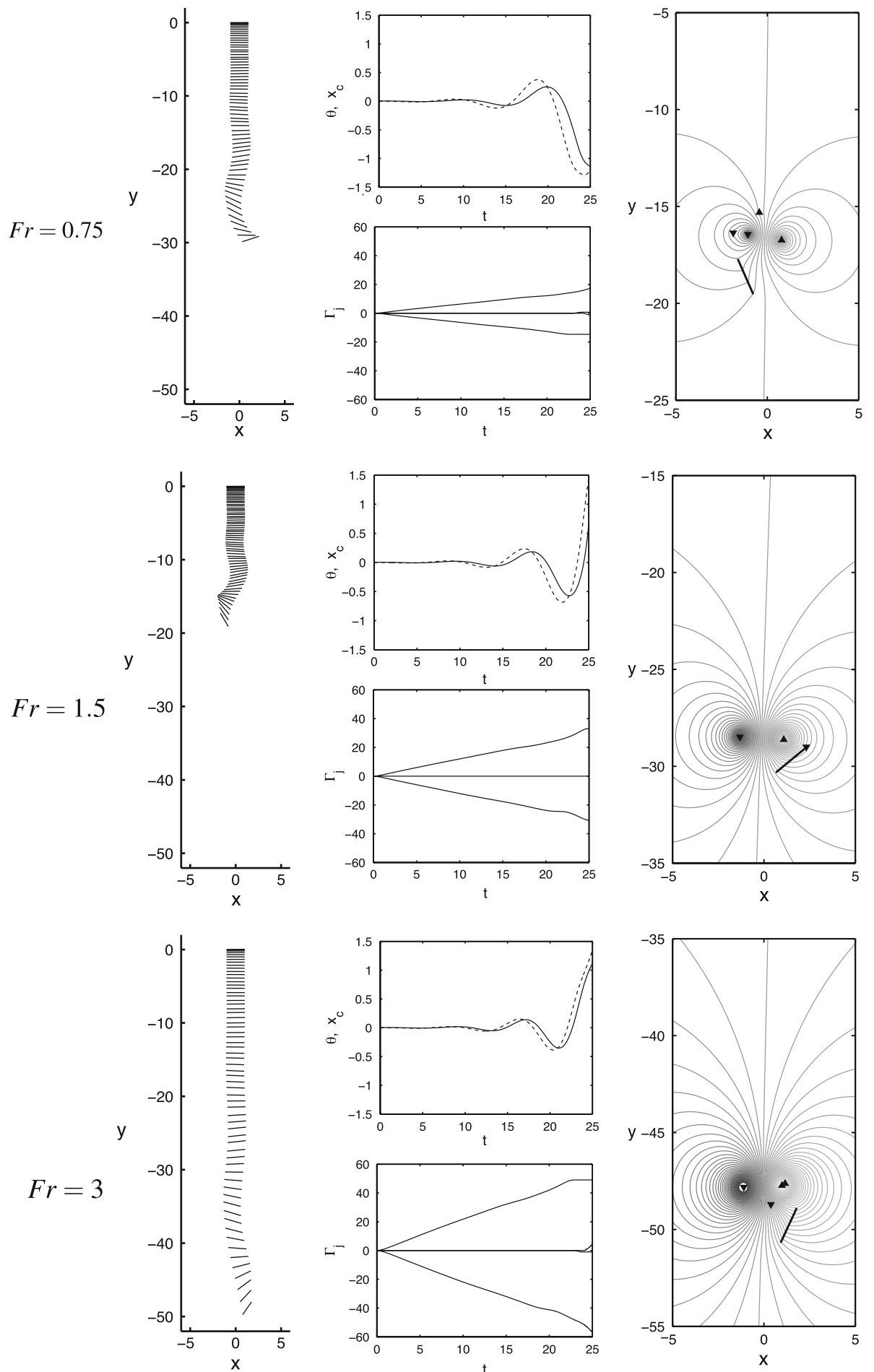


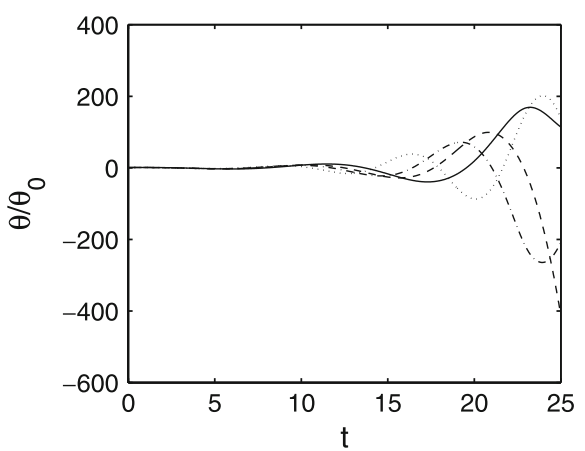

(a)

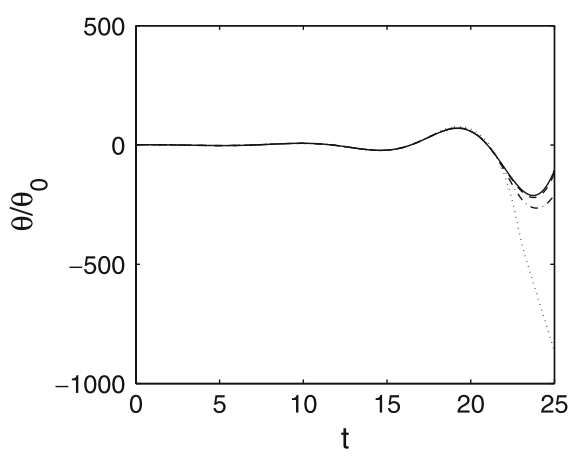

(b)

Fig. 8 Evolution of $\theta / \theta_{0}$ in time. a Influence of $F r: \theta / \theta_{0}$ is plotted for $\theta_{0}=\pi / 1,024$ and $F r=0.2$ (solid), $F r=0.5$ (dashed), $F r=1$ (dash-dotted) and $F r=5$ (dotted). b Influence of $\theta_{0}: \theta / \theta_{0}$ is plotted for $F r=1$ and $\theta_{0}=\pi / 4,096$ (solid), $\theta_{0}=\pi / 2,048$ (dashed), $\theta_{0}=\pi / 1,024$ (dash-dotted) and $\theta_{0}=\pi / 512$ (dotted)

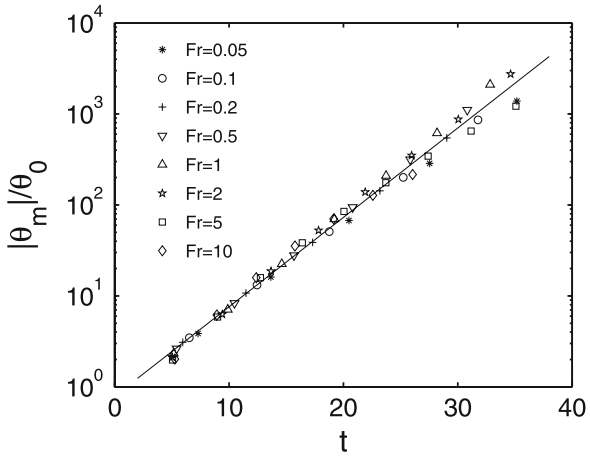

(a)

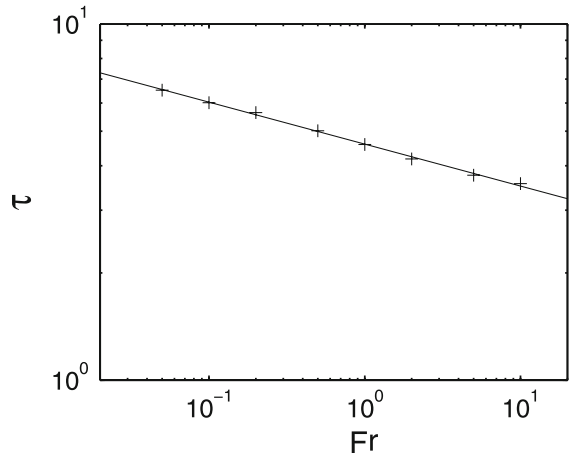

(b)

Fig. 9 Influence of $F r$ on the development of the instability for $\theta_{0}=\pi / 8,092$. a Successive maxima of $|\theta| / \theta_{0}$. The solid line corresponds to the best exponential fit. The corresponding grow rate is about 0.23 . b Pseudo-period $\tau$ of the growing oscillations. The solid line shows the best power-law fit corresponding to $\mathrm{Fr}^{-0.12}$

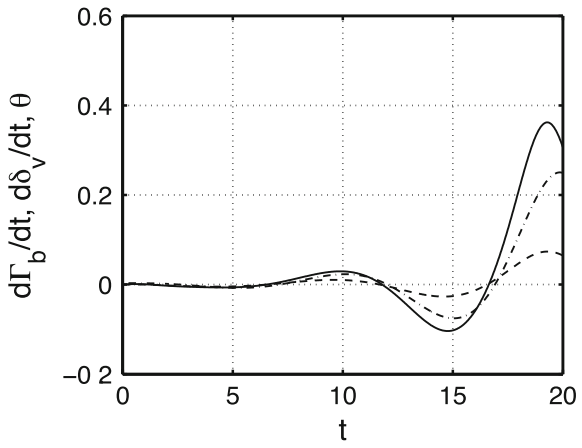

(a)

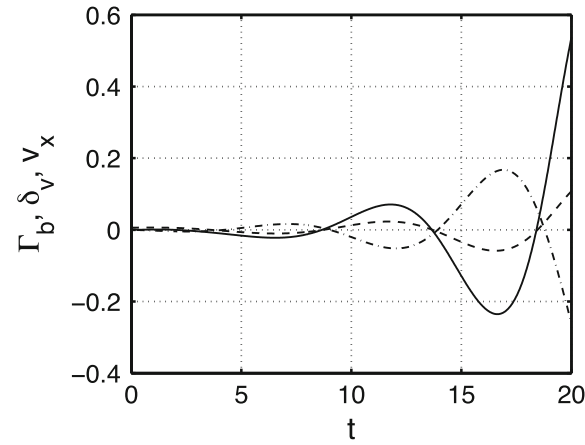

(b)

Fig. 10 a Evolution of the rate of change of the circulation around the body $\dot{\Gamma}_{b}$ (solid), the relative vertical velocity of the vortices $\dot{\delta}_{v}$ (dashed) and the orientation of the plate $\theta$ (dash-dotted) in the fluttering regime for $F r=0.75$ and $\theta_{0}=\pi / 1,024$. b Evolution of the circulation around the body $\Gamma_{b}$ (solid), the relative vertical distance of the vortices $\delta_{v}$ (dashed) and the horizontal plate velocity $v_{x}$ (dash-dotted) in the fluttering regime for $F r=0.75$ and $\theta_{0}=\pi / 1,024$ 


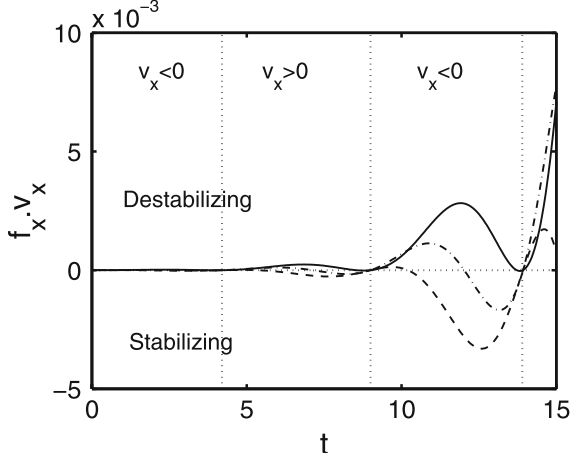

(a)

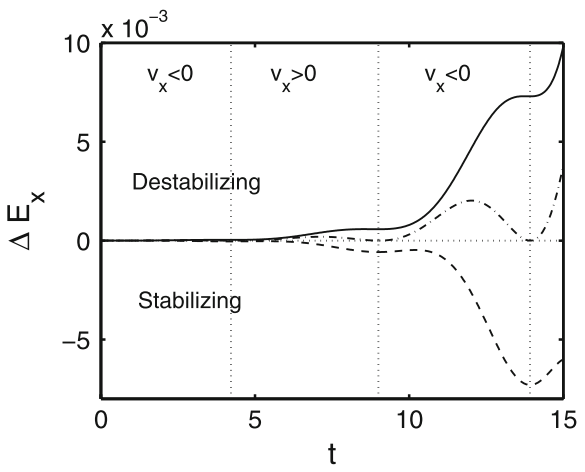

(c)

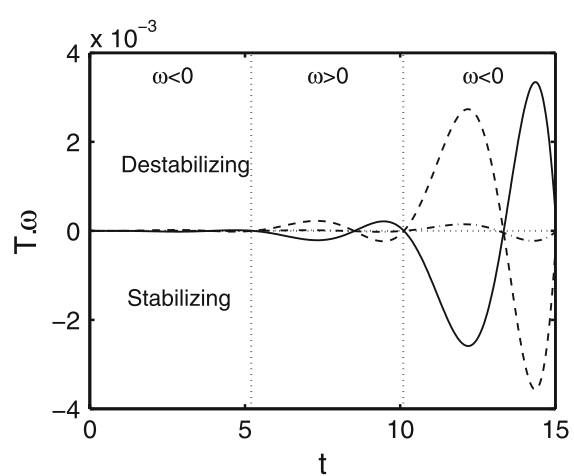

(b)

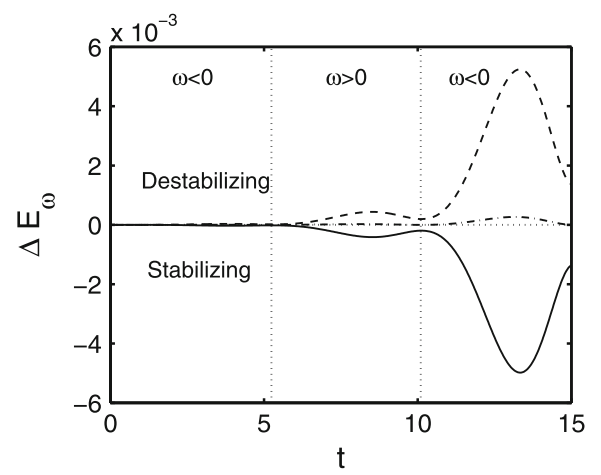

(d)

Fig. 11 Stabilizing and destabilizing effects of the fluid on the falling card for $F r=0.75$ and $\theta_{0}=\pi / 1,024$. Top Rate of work of the vortex contribution (solid), of the added inertia contribution (dashed) and of the total (dash-dotted) horizontal force (a) and torque (b) on the falling card. Bottom Horizontal (c) and rotational (d) kinetic energy gain of the plate due to the vortices (solid) and to the added inertia contribution (dashed). The horizontal and rotational solid kinetic energy is also plotted (dash-dotted). The sign of the horizontal velocity $v_{x}=\operatorname{Re}(\dot{c})$ and angular velocity $\omega$ is indicated for reference

to the solid body. Since the body is rigid, these two quantities are easily related. As discussed in Sect. 3.4.3, the fluid effects can be decomposed in two parts: added inertia and transfer of linear and angular momentum to the vortices.

The equations of motion of the plate can be rewritten as

$$
2 F r \ddot{c}=-2 \mathrm{i} F r+f^{a}+f^{v}, \quad \frac{2 F r}{3} \ddot{\theta}=\mathcal{T}^{a}+\mathcal{T}^{v},
$$

where the first term on the right-hand side of Newton's second law is the gravity forcing and $a$ and $v$ superscripts refer to forces and torques due to added inertia and transfer of momentum to the vortices, respectively. The vertical motion is at leading order identical to the broadside-on case, so we focus on the horizontal and angular motions of the plate.

The effects of vortex shedding and added inertia on the horizontal and angular motions of the plate are analysed in Fig. 11 by plotting the rate of work of the different contributions as well as its integrated effect in time (i.e., the change of solid kinetic energy due to the corresponding force or torque). A positive rate of work will be destabilizing: the forcing acts in the same direction as the motion. Figure 11a, c shows that added inertia tends to dampen the horizontal side-to-side motion of the card, while the transfer of momentum to the vortices has a destabilizing effect and creates a net energy gain. In the angular motion, the effects of added inertia and of the vortices give opposite contributions to the rotational work $\mathcal{T} \omega$ on the plate, the former being slightly larger than the latter (Fig. 11b, d).

The transfer of horizontal momentum to the vortices is therefore at the origin of the destabilization of the broadside-on fall and arises from the asymmetry of the wake. We now propose a physical analysis of the origin of this destabilizing horizontal momentum transfer. For a small enough initial angle, the fall of the card is a perturbation of the symmetric case studied in Sect. 5.2, with a pair of counter-rotating vortices, with intensity 


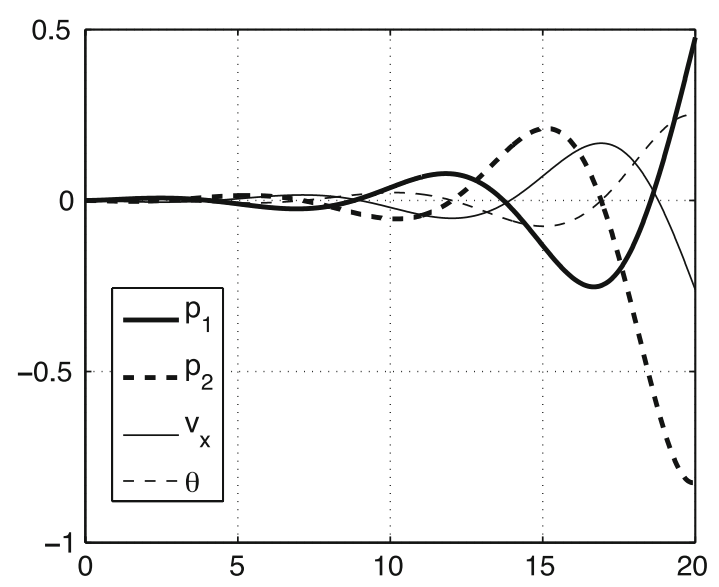

Fig. 12 Evolution for $\theta_{0}=\pi / 1,024$ and $F r=0.75$ of the different contributions to the horizontal momentum associated with the vortices $p_{1}$ (thick-solid) and $p_{2}$ (thick-dashed) as defined in (39). The plate's horizontal velocity $v_{x}$ (thin solid) and orientation $\theta$ (thin-dashed) are also plotted for comparison

and position in the mapped plane $\left(\Gamma_{0}, \zeta_{0}\right)$ and $\left(-\Gamma_{0},-\bar{\zeta}_{0}\right)$. The vertical physical distance between the plate and the vortex pair, defined in Sect. 5.2, is then $d_{0}^{(v)}=\zeta_{0}+a^{2} / \zeta_{0}$.

From Sect.3.4.3 and [26], the horizontal linear momentum of the vortices is obtained at leading order as

$$
p_{x}^{(v)} \sim \overbrace{\Gamma_{0} \delta_{v}-\Gamma_{b} d_{0}^{(v)}}^{p_{1}} \overbrace{-4 \theta \Gamma_{0} \operatorname{Re}\left(\frac{a^{2}}{\zeta_{0}}\right)}^{p_{2}} .
$$

The two terms on the right-hand side have the following interpretations:

- $p_{1}$ is the horizontal momentum due to the asymmetry of the wake: if $\delta_{v}>0$, the intrinsic velocity of the vortex pair is tilted to the right and the wake carries positive horizontal momentum. In the same way, if $\Gamma_{1}>\left|\Gamma_{2}\right|\left(\Gamma_{b}<0\right)$ the asymmetry in the intensity of the vortices deflects the wake slightly to the right, and corresponds to a positive horizontal vortex momentum. As $\delta_{v}$ and $\Gamma_{b}$ vary in phase (see Fig. 10b), the first two terms in (39) have opposite effects. $p_{1}$ is observed to vary in a very good approximation like $-v_{x}$, with $v_{x}$ the horizontal velocity of the plate's center of mass (Fig. 12). The corresponding force $-\dot{p}_{1}$ therefore behaves like a (negative) correction to the plate's inertia (not to be mistaken to the added inertia discussed in Sect. 5.3). In particular, it tends to accelerate the plate's horizontal motion at the beginning of each half-period when $\left|v_{x}\right|$ starts increasing.

- $\quad p_{2}$ is the horizontal momentum associated with the image vorticity and is due to the non-horizontal orientation of the plate: $\Gamma_{0} \operatorname{Re}\left(a^{2} / \zeta_{0}\right)$ corresponds to the vortex momentum normal to the plate associated with the image vorticity and is monotonically increasing in time. To a very good approximation, $p_{2}$ varies in phase with $-\theta$ (Fig. 12): for $\theta<0$ and a symmetric vortex pair, the vortices would be deflected to the right, thereby inducing a positive horizontal vortex momentum. The corresponding force on the plate $-\dot{p}_{2}$ therefore behaves like $\omega$. As the horizontal and angular motions are almost in phase (with the latter being slightly in advance on the former), the vortex force associated with the rotation of the plate around its vortex wake is acting in the same direction as the plate's velocity throughout most of the oscillation.

In a summary, during the fluttering oscillations of the plate, the relative motions of the plate and of the vortices, and particularly the resulting deflection of vortex momentum associated with the image vorticity (similar to the behavior of point vortices close to a wall), generates a destabilizing force that cannot be damped by added inertia effects. This destabilization is transmitted to the rotational motion of the plate through added inertia.

\section{Conclusions}

A general framework for the study of the two-dimensional coupled motions of a sharp-edged solid body and a high-Re flow has been presented using potential flow theory and a representation of the vortical wake in terms of 
point vortices with unsteady intensity determined to satisfy the regularity of the flow velocity on the solid body. The Brown-Michael vortex model, used previously to study vortex shedding past a fixed semi-infinite or finite plate, has been extended to arbitrary body shapes and coupled to the solid dynamics to provide a reduced-order description of the fluid-solid problem in terms of ordinary differential equations only. The forces and torques on the solid body have been analysed in terms of transfer of linear and angular momentum to the vortices. This physically based model significantly reduces the computational cost and complexity of the simulation of the fluid-solid problem compared to direct numerical simulations and also compared to a continuous vortex sheet approach. In addition, the numerical and physical difficulties of the vortex sheet model, due to the necessary smoothing of the Cauchy kernel to regularize the free vortex sheet velocity and to the treatment of the last Lagrangian vortex element, are not present here. The point vortex approach described here also provides a powerful tool for understanding the structure of the vortical wake and its influence on the solid body.

The method was then used in the simple geometry of a flat plate to study the fall of a rigid card in a fluid initially at rest. The different forces and torques applied by the fluid on the card were analysed. The broadsideon fall, where the plate falls in its horizontal position, was found to be unstable and, for small initial angles, a fluttering regime with growing amplitude was observed. This is in agreement with numerous experimental and numerical studies at high $R e$ present in the literature. Using the reduced-order representation of the vortex wake provided by the point vortex model, it was shown that the destabilization is caused by the deflection of linear momentum associated with the vortices by the plate's fluttering motion around them. The net effect is an amplification of the horizontal motion which then amplifies the rotation amplitude of the fluttering motion through the coupling of rotation and translation by added inertia. In comparison, a representation of the fluid effects exclusively in terms of added inertia was found unable to predict such a behavior. This example shows the physical validity of the proposed model as well as the interest of a low order representation to understand the destabilization mechanism. The point vortex method was recently used to study the coupling between flexible bodies and high $R e$ flows [37].

Further integration of the coupled equations of motion showed different possible regimes, including the flip of the card once the $\theta$ oscillations reach a finite amplitude, and the transition to rotating patterns that can be identified to the tumbling regimes observed experimentally. This is an improvement compared to the vortex sheet approach, in which the shedding failure occured always before the flip of the card. The integration eventually breaks down at an unfavorable shedding event, limiting our ability to study the influence of $\theta_{0}$ and $\mathrm{Fr}$ on the long-time regime.

The unsteady point vortex method is therefore limited, as is the vortex sheet method, by its inability to represent vortices that are not shed away from the solid body. Failure events were identified when a new vortex is being shed under unfavorable conditions, where the outside flow tends to push the newly shed vortex back onto the shedding corner. This situation occurs mostly in leading-edge shedding conditions and is inherent to the inviscid representation chosen here. Several solutions could be considered including the shedding of multiple vortices at the same time, or the absence of shedding of a new vortex: under such conditions, the flow velocity is singular at the shedding edge, which has a non-zero bound vorticity, representing the attached vortex. The shedding of a vortex from that corner would then be restarted at a later time when the conditions are favorable.

Despite this limitation, the point vortex method presented here is a powerful tool in a large variety of situations where leading-edge shedding is not present or is neglected. Its computational simplicity makes it suitable for a large number of situations where the cost of direct numerical simulation is prohibitive (e.g. optimization problems).

Acknowledgments This work was funded by NSF award CTS-0133978. We are grateful to Professor J. B. Keller for introducing us to this problem and to Professors H. K. Cheng and D. I. Pullin for helpful conversations. We are also grateful to the referees for their helpful comments.

Open Access This article is distributed under the terms of the Creative Commons Attribution Noncommercial License which permits any noncommercial use, distribution, and reproduction in any medium, provided the original author(s) and source are credited.

\section{Appendix A: Brown-Michael equation: conservation of the fluid momentum around the vortex and branch cut}

In this section we provide a conservation of momentum argument for the Brown-Michael equation (1) based on complex analysis. This is essentially a formalization of the physical arguments of [9] written in the lan- 


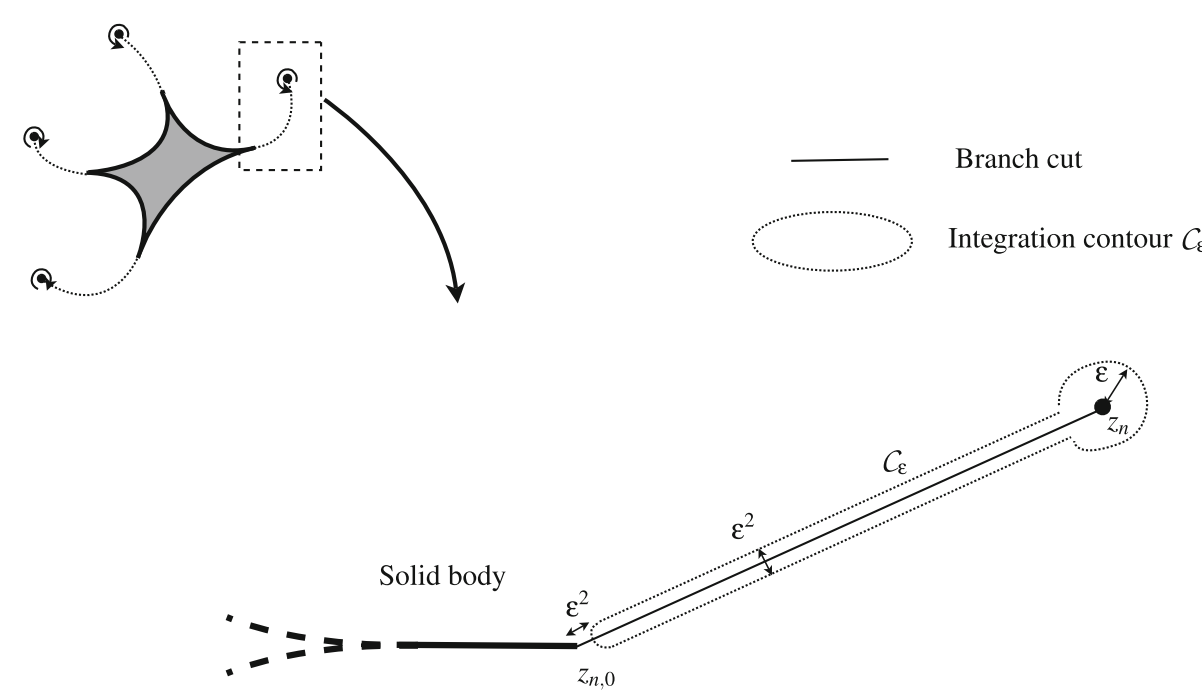

Fig. 13 Conservation of momentum around the unsteady point vortex and its corresponding branch cut

guage of the present work (see also [45]). For a general contour $C$ enclosing only fluid and moving with a (spatially-dependent) velocity $\mathbf{u}_{c}$, Newton's second law for the fluid inside $C$ is given by

$$
\dot{\mathbf{M}}=-\int_{C} p \mathbf{n} \mathrm{d} l-\int_{C} \rho \mathbf{u}\left[\left(\mathbf{u}-\mathbf{u}_{c}\right) \cdot \mathbf{n}\right] \mathrm{d} l,
$$

where the left-hand side is the rate of change of the momentum in $C$ and the terms on the right-hand side are respectively the force applied by the outside fluid on the contour and the flux of momentum through $C$. Using complex notation, $w=u-\mathrm{i} v$, and Bernoulli's equation $p=p_{0}(t)-\rho\left(F_{t}+\bar{F}_{t}+w \bar{w}\right) / 2$, (40) simplifies to

$$
\dot{M}=-\frac{\mathrm{i} \rho}{2} \int_{C}\left(F_{t}+\bar{F}_{t}\right) \mathrm{d} z+\overline{\frac{\mathrm{i} \rho}{2} \int_{C} w\left(w-w_{c}\right) \mathrm{d} z}+\overline{\frac{\mathrm{i} \rho}{2} \bar{w}_{c} \int_{C} w \mathrm{~d} \bar{z}} .
$$

When shedding an unsteady vortex from corner $n$, the logarithmic form of the complex potential leads to a branch cut between $z_{n, 0}$ and $z_{n}$. We choose here to define it as the straight line connecting these two points (but its detailed location is arbitrary). We now consider a contour $\mathcal{C}_{\epsilon}$ enclosing the vortex and the branch cut as shown on Fig. 13. As $\epsilon$ goes to zero, $\mathcal{C}_{\epsilon}$ encloses only the vortex and the branch cut.

The chosen contour crosses the branch cut. This is not problematic as we are not carrying out an inverse Fourier transform or other integral that requires avoiding branch cuts. We are integrating a function whose real part is discontinuous, and this is why physically there is a net force. In (41), the last two integrals only involve $w$, a single-valued function analytic everywhere except at the vortex position. The intensity of the vortex is chosen to satisfy the regularity condition so that $w$ is bounded at the generating corner $z_{n, 0}$. The existence of the branch cut only affects the first integral. The integrand $\operatorname{Re}(F)$ is discontinuous across the branch cut, but this discontinuity is finite and can be proved to be equal to $\pm \Gamma_{n}$.

Note that [21] uses a contour enclosing all the vortices and branch cuts as well as the plate. This contour can be used to derive an expression for the force on a solid from the expansion of the potential at infinity of the same form as the one considered in this paper, but uses the Brown-Michael equation (1) to obtain this result. In particular only one contour is used, which cannot lead to separate equations for each unsteady vortex.

A careful calculation shows that

$$
\int_{C_{\epsilon}} \operatorname{Re}\left[-\mathrm{i} \log \left(z-z_{n}\right)\right] \mathrm{d} z=-2 \pi\left(z_{n}-z_{n, 0}\right)+O(\epsilon) .
$$

The complex potential and velocity can be decomposed as

$$
F=\frac{\Gamma_{n}}{2 \mathrm{i} \pi} \log \left(z-z_{n}\right)+\tilde{F}_{n}(z), \quad w=\frac{\Gamma_{n}}{2 \mathrm{i} \pi\left(z-z_{n}\right)}+\tilde{w}_{n}(z)
$$


with $\tilde{F}_{n}$ and $\tilde{w}_{n}$ single-valued and analytic on and inside $\mathcal{C}_{\epsilon}$ except at the vortex position $z_{n}$. Near the vortex, the velocity of the contour is $w_{c}=\dot{\bar{z}}_{n}$. Using these results, the integrals in (41) can be evaluated exactly and when $\epsilon \rightarrow 0$, we obtain

$$
\dot{M} \rightarrow \mathrm{i} \rho\left[\dot{\Gamma}_{n}\left(z_{n}-z_{n, 0}\right)+\Gamma_{n}\left(\dot{z}_{n}-\overline{\tilde{w}}_{n}\right)\right] .
$$

However, when $\epsilon \rightarrow 0$, the surface enclosed in $\mathcal{C}_{\epsilon}$ goes to zero and the velocity is bounded along the branch cut. Near the vortex, the flow is purely azimuthal so when the contour is shrinking down, the linear momentum enclosed in $\mathcal{C}_{\epsilon}$ goes to zero, leading to the Brown-Michael equation (1).

The derivation detailed here shows that the Brown-Michael equation leads to Newton's second law being satisfied in the fluid around the vortex and around the branch cut. Any choice of contour $C$ enclosing only part of the branch cut would have led to a similar equation

$$
\dot{z}_{n}+\left(z_{n}-z^{*}\right) \frac{\dot{\Gamma}_{n}}{\Gamma_{n}}=\bar{w}_{n}
$$

with $z^{*}$ the position of the crossing point of $C$ with the branch cut. Kirchhoff's equation corresponds to $z^{*}=z_{n}$ and only conserves the fluid momentum around the vortex. However, only the choice $z^{*}=z_{n, 0}$ corresponding to the Brown-Michael equation (1) guarantees the conservation of momentum in an integrated sense around the whole branch cut. If $z^{*} \neq z_{n, 0}$, there exists an unbalanced force on the remainder of the branch cut between $z_{n, 0}$ and $z^{*}$. Nothing in this argument depends on the shape of the body.

\section{References}

1. Andersen, A., Pesavento, U., Wang, Z.J.: Analysis of transitions between fluttering, tumbling and steady descent of falling cards. J. Fluid Mech. 541, 91-104 (2005)

2. Andersen, A., Pesavento, U., Wang, Z.J.: Unsteady aerodynamics of fluttering and tumbling plates. J. Fluid Mech. 541, 65-90 (2005)

3. Belmonte, A., Eisenberg, H., Moses, E.: From flutter to tumble: inertial drag and Froude similarity in falling paper. Phys. Rev. Lett. 81, 345-348 (1998)

4. Belmonte, A., Moses, E.: Flutter and tumble in fluids. Phys. World. Mag. 12, 21-25 (1999)

5. Bergou, A., Xu, S., Wang, Z.J.: Passive wing pitch reversal in insect flight. J. Fluid Mech. 591, 321-337 (2007)

6. Berman, G., Wang, Z.J.: Energy-minimizing kinematics in hovering insect flight. J. Fluid Mech. 582, 153-168 (2007)

7. Borisov, A.V., Kozlov, V.V., Mamaev, I.S.: Asymptotic stability and associated problems of dynamics of falling rigid body. Reg. Chaotic Dyn. 12, 531-565 (2007)

8. Borisov, A.V., Mamaev, I.S., Ramodanov, S.M.: Dynamic interaction of point vortices and a two-dimensional cylinder. J. Math. Phys. 48, 065403 (2007)

9. Brown, C.E., Michael, W.H.: Effect of leading edge separation on the lift of a delta wing. J. Aero. Sci. 21, 690-694 \& 706 (1954)

10. Cheng, H.K.: Remarks on non linear lift and vortex separation. J. Aero. Sci. 19, 212-214 (1954)

11. Clements, R.R.: An inviscid model of two-dimensional vortex shedding. J. Fluid Mech. 57, 321-336 (1973)

12. Cortelezzi, L.: A theoretical and computational study on active wake control. Ph.D. thesis, California Institute of Technology (1993)

13. Cortelezzi, L.: On the unsteady separated flow past a semi-infinite plate. exact solution of the brown and michael model, scaling and universality. Phys. Fluids 7, 526-529 (1995)

14. Cortelezzi, L.: Nonlinear feedback control of the wake past a plate with a suction point on the downstream wall. J. Fluid Mech. 327, 303-324 (1996)

15. Cortelezzi, L., Chen, Y.C., Chang, H.L.: Nonlinear feedback control of the wake past a plate: from a low-order model to a higher-order model. Phys. Fluids 9, 2009-2022 (1997)

16. Cortelezzi, L., Leonard, A.: Point vortex model of the unsteady separated flow past a semi-infinite plate with transverse motion. Fluid Dyn. Res. 11, 263-295 (1993)

17. Crowdy, D.G., Surana, A., Yick, K.Y.: The irrotational motion generated by two planar stirrers in inviscid fluid. Phys. Fluids 19, 018103 (2007)

18. Edwards, R.H.: Leading-edge separation from delta wings. J. Aero. Sci. 21, 134-135 (1954)

19. Field, S.B., Klaus, M., Moore, M.G., Nori, F.: Chaotic dynamics of falling disks. Nature 388, 252-254 (1997)

20. Graham, J.M.R.: Vortex shedding from sharp edges. Imperial College Aero. Rep. 77-06 (1977)

21. Graham, J.M.R.: The forces on sharp-edged cylinders in oscillatory flow at low Keulegan-Carpenter numbers. J. Fluid Mech. 97, 331-346 (1980)

22. Howe, M.S.: Emendation of the Brown and Michael equation, with application to sound generation by a vortex motion near a half-plane. J. Fluid Mech. 329, 89-101 (1996)

23. Huang, J.Y: Moving coordinates methods and applications to the oscillations of a falling slender body. In: Šarler, B., Brebbia, C.A. (eds.) Moving Boundaries VI: Computational Modelling of Free and Moving Boundary Problems, pp. 73-82. WIT Press, Southampton (2001) 
24. Jones, M.A.: The separated flow of an inviscid fluid around a moving plate. J. Fluid Mech. 496, 405-441 (2003)

25. Jones, M.A., Shelley, M.J.: Falling cards. J. Fluid Mech. 540, 393-425 (2005)

26. Kanso, E., Oskouei, B.G.: Stability of a coupled body-vortex system. J. Fluid Mech. 600, 77-94 (2008)

27. Keller, J.B.: Vortex sheets as vortices in two-dimensional flow around a body (1998, unpublished)

28. Kozlov, V.V.: Heavy rigid body falling in an ideal fluid. Mech. Solids 24, 9-17 (1989)

29. Kozlov, V.V.: On the problem of a heavy rigid body falling in a resisting medium. Vestnik Moskow Univ. Ser. I Mat. Meckh. 1, 79-86 (1990)

30. Krasny, R.: Desingularisation of periodic vortex sheet roll-up. J. Comput. Phys. 65, 292-313 (1986)

31. Lamb, H.: Hydrodynamics, 6th edn. Dover, New York (1932)

32. Lin, C.C.: On the motion of vortices in two dimensions. Proc. Natl. Acad. Sci. 27, 570-575 (1941)

33. Llewellyn Smith, S.G., Michelin, S., Crowdy, D.G.: The dipolar field of rotating bodies in two dimensions. J. Fluid Mech. 607, 109-118 (2008)

34. Mahadevan, L.: Tumbling of a falling card. C. R. Acad. Sci. Ser. IIb 323, 729-736 (1996)

35. Mahadevan, L., Aref, H., Jones, S.W.: Comment on 'behavior of a falling paper' (with reply). Phys. Rev. Lett. 75, 1420 (1995)

36. Maxwell, J.C.: On a particular case of the decent of a heavy body in a resisting medium. Camb. Dublin Math. J. 9, 145$148(1854)$

37. Michelin, S., Llewellyn Smith, S.G., Glover, B.J.: Vortex shedding model of a flapping flag. J. Fluid Mech. 617, 1-10 (2008)

38. Milne-Thomson, L.M.: Theoretical Hydrodynamics. Macmillan, New York (1968)

39. Mittal, R., Seshadri, V., Udaykumar, H.S.: Flutter, tumble and vortex induced autorotation. Theor. Comput. Fluid Dyn. 17, $165-170$ (2004)

40. Newman, J.N.: Marine Hydrodynamics. MIT Press, Cambridge (1977)

41. Pesavento, U., Wang, Z.J.: Falling paper: Navier-Stokes solutions, model of fluid forces, and center of mass elevation. Phys. Rev. Lett. 93, 144501 (2004)

42. Pullin, D.I.: The large-scale structure of unsteady self-similar rolled-up vortex sheets. J. Fluid Mech. 88, 401-430 (1978)

43. Ramodanov, S.M.: Motion of a circular cylinder and a vortex in an ideal fluid. Reg. Chaotic Dyn. 6, 33-38 (2000)

44. Rott, N.: Diffraction of a weak shock with vortex generation. J. Fluid Mech. 1, 111-128 (1956)

45. Saffman, P.G.: Vortex Dynamics. Cambridge University Press, London (1992)

46. Sedov, L.I.: Two-Dimensional Problems in Hydrodynamics and Aerodynamics. Interscience, New York (1965)

47. Shashikanth, B.N.: Poisson brackets for the dynamically interacting system of a $2 \mathrm{D}$ rigid cylinder and N point vortices: the case of arbitrary smooth cylinder shapes. Reg. Chaotic Dyn. 10, 1-14 (2005)

48. Shashikanth, B.N., Marsden, J.E., Burdick, J.W., Kelly, S.D.: The hamiltonian structure of a 2D rigid circular cylinder interacting dynamically with N point vortices. Phys. Fluids 14, 1214-1227 (2002)

49. Shukla, R.K., Eldredge, J.D.: An inviscid model for vortex shedding from a deforming body. Theor. Comput. Fluid Dyn. 21, 343-368 (2007)

50. Smith, E.H.: Autorotating wings: an experimental investigation. J. Fluid Mech. 50, 513-534 (1971)

51. Smith, J.H.B.: Improved calculations of leading edge separation from slender delta wings. Proc. R. Soc. A 306, 67-90 (1968)

52. Tanabe, Y., Kaneko, K.: Behavior of a falling paper. Phys. Rev. Lett. 73, 1372-1375 (1994)

53. Tanabe, Y., Kaneko, K.: Tanabe and Kaneko reply. Phys. Rev. Lett. 75, 1421 (1995)

54. Triantafyllou, M.S., Triantafyllou, G.S., Yue, D.K.P.: Hydrodynamics of fishlike swimming. Ann. Rev. Fluid Mech. 32, $33-53(2000)$

55. Wang, Z.J.: Two dimensional mechanism for insect hovering. Phys. Rev. Lett. 85, 2216-2219 (2000)

56. Wang, Z.J.: Dissecting insect flight. Annu. Rev. Fluid Mech. 37, 183-210 (2005)

57. Willmarth, W.W., Hawk, N.E., Harvey, R.L.: Steady and unsteady motions and wakes of freely falling disks. Phys. Fluids 7, 197-208 (1964)

58. Zhu, L.: Viscous flow past a flexible fibre tethered at its center point: vortex shedding. J. Fluid Mech. 587, 217-234 (2007)

59. Zhu, L., Peskin, C.: Simulation of flapping flexible filament in a flowing soap film by the immersed boundary method. J. Comput. Phys. 179, 452-468 (2002) 